\title{
The Dynamics of Stochastic Volatility: Evidence from Underlying and Options Markets
}

\author{
Christopher S. Jones* \\ Simon School of Business \\ Carol Simon Hall 3-110P \\ University of Rochester \\ Rochester, NY 14627 \\ Tel: (716) 275-3491 \\ Fax: (716) 273-1140 \\ c_s_jones@simon.rochester.edu \\ http://www.simon.rochester.edu/fac/c_s_jones
}

First draft: October 1999

This revision: December 2000

\begin{abstract}
This paper proposes and estimates a more general parametric stochastic variance model of equity index returns than has been previously considered using data from both underlying and options markets. The parameters of the model under both the objective and riskneutral measures are estimated simultaneously. I conclude that the square root stochastic variance model of Heston (1993) and others is incapable of generating realistic returns behavior and find that the data are more accurately represented by a stochastic variance model in the CEV class or a model that allows the price and variance processes to have a time-varying correlation. Specifically, I find that as the level of market variance increases, the volatility of market variance increases rapidly and the correlation between the price and variance processes becomes substantially more negative. The heightened heteroskedasticity in market variance that results generates realistic crash probabilities and dynamics and causes returns to display values of skewness and kurtosis much more consistent with their sample values. While the model dramatically improves the fit of options prices relative to the square root process, it falls short of explaining the implied volatility smile for shortdated options.
\end{abstract}

\section{JEL classification: G12, C11.}

Keywords: continuous-time estimation, stochastic volatility, option pricing, Bayesian analysis, stock market crashes.

\footnotetext{
*I am grateful to Greg Bauer, Mike Chernov, Mike Johannes, Jun Pan, Neil Shephard, Ane Tamayo, and seminar participants at McGill, Rochester, the 2000 Meetings of the Western Finance Association, the 2000 World Congress of the Econometric Society, and the 2000 Duke-CIRANO Conference on Risk Neutral and Objective Probability Distributions for many helpful comments, to Iulian Obreja for sharing his technical expertise, and to George Tauchen and Nour Meddahi for two very helpful discussions of the paper. All errors remain my own.
} 


\section{The Dynamics of Stochastic Volatility: Evidence from Underlying and Options Markets}

This paper proposes and estimates a more general parametric stochastic variance model of equity index returns than has been previously considered using data from both underlying and options markets. The parameters of the model under both the objective and risk-neutral measures are estimated simultaneously. I conclude that the square root stochastic variance model of Heston (1993) and others is incapable of generating realistic returns behavior and find that the data are more accurately represented by a stochastic variance model in the CEV class or a model that allows the price and variance processes to have a time-varying correlation. Specifically, I find that as the level of market variance increases, the volatility of market variance increases rapidly and the correlation between the price and variance processes becomes substantially more negative. The heightened heteroskedasticity in market variance that results generates realistic crash probabilities and dynamics and causes returns to display values of skewness and kurtosis much more consistent with their sample values. While the model dramatically improves the fit of options prices relative to the square root process, it falls short of explaining the implied volatility smile for short-dated options. 


\section{Introduction}

The dynamic nature of asset price volatility has long been a popular subject in the empirical finance literature, and even greater interest in volatility dynamics has followed the development of stochastic volatility models of option pricing. These models attribute the higher prices of options far from "at-the-money" to return non-normality generated by time-varying volatility. Because much of the interest in options focuses on the options that are furthest from atthe-money and therefore have positive payoffs only in extremely rare circumstances, precise characterizations of volatility dynamics are particularly important.

While the need to better model extreme events for the purposes of option pricing made the task of understanding volatility more exacting, the existence of options has made the researcher's data set much richer, so that more finely-tuned models may be investigated with greater hope of obtaining accurate results.

Traditionally, volatility dynamics in asset prices have been explored using the time series of returns on the assets being studied. Following the work of Engle (1982) and Bollerslev (1986), models in the ARCH class have been studied extensively for this purpose and remain popular today. Perhaps because of the rapid growth in derivatives markets, continuous-time stochastic volatility models have recently become popular as well, and a growing body of research is concerned with fitting these models to asset returns. Two recent examples are the papers by Gallant and Tauchen (1997) and Andersen, Benzoni, and Lund (2000).

An alternative approach is to infer the risk-neutralized volatility dynamics from the prices of options. While methods of inferring risk-neutral returns probabilities from the cross section of options prices are well-known following the seminal work of Breeden and Litzenberger (1978), more recent studies such as Bates (2000) and Bakshi, Cao, and Chen (1997) fit parametric stochastic volatility models to option prices to learn about the parameters of these models.

But since the risk-neutral and objective measures are not wholly dissimilar, there must be an advantage to using both sets of data (the time series of the underlying's prices and the prices of options on it) to infer both measures simultaneously, a point made forcefully by Chernov and Ghysels (2000). In addition, since it is the difference between the two measures that defines risk premia, it may further be advantageous to estimate the two measures together so that estimation errors relating to these differences can be treated in a sensible way. In fact,

a number of recent papers have pursued this idea. Pan (2000), Poteshman (1998), and Chernov 
and Ghysels (2000) all use some combination of a time series of the underlying price and one or more time series of liquid, short-term, near-the-money option prices to infer the dynamics of the underlying volatility process under both the objective and risk-neutral measures. ${ }^{1}$

In this paper, I consider a more general form of stochastic variance model than has been considered previously and estimate the model in a Bayesian framework from a bivariate time series of returns and options prices. The analysis results in parameter estimates for both the objective and risk-neutral processes. The results show that the model I propose generates more realistic dynamics for stochastic variance and for equity index returns than does the standard square root model used by Heston (1993) and many others. I find that as variance increases, its own volatility increases at an even faster rate, and the correlation between prices and instantaneous variance also becomes significantly more negative. A positive shock to market variance therefore increases the probability that market variance may grow even larger. The compound effect of several of these shocks can be large, and extreme returns may result. Because the price and variance processes are negatively correlated, particularly in high volatility states, this extreme return will more often than not be negative, frequently of a magnitude that the square root process is incapable of generating.

I focus on the implications of these models for rare events, since many of the outstanding puzzles in options markets and other areas of empirical asset pricing depend primarily on the likelihood and nature of such events. I find a realistic degree of non-normality implied by the new model, while the higher moments generated by the square root model fall far short of the levels observed in actual data. The implications for stock market crashes that are generated by the new model are also surprisingly accurate. Model-implied options prices, however, while greatly improved over the square root specification, still fail to match observed prices of shortdated options.

Section 2 of this paper motivates the econometric specification and outlines a few of its properties. The estimation method used in the paper is described in section 3. Section 4 contains the results, including parameter estimates and the models' implications for higher moments in returns, option pricing, and the existence and nature of stock market crashes. Section 5 summarizes and discusses the results.

\footnotetext{
${ }^{1}$ Studies by Benzoni (1999) and Jiang and van der Sluis (1998) have similar objectives, but both use options data only to estimate the parameters that are not identified by data on the underlying. Additional literature is reviewed in Chernov and Ghysels (1999).
} 


\section{The model}

By far the most popular recent formulations of stochastic volatility in continuous time have been variants of the stochastic variance model of Heston (1993). Using a square root process to represent the dynamics of instantaneous variance, Heston assumes that returns are generated by

$$
\begin{aligned}
& d S_{t}=\mu S_{t} d t+\sqrt{V_{t}} S_{t} d Z_{t}^{(1)} \\
& d V_{t}=\left(\alpha+\beta V_{t}\right) d t+\sigma \sqrt{V_{t}} d Z_{t}^{(2)}
\end{aligned}
$$

where $d Z_{t}^{(1)}$ and $d Z_{t}^{(2)}$ are increments of Brownian motion with correlation $\rho d t$. Heston demonstrates how Fourier inversion techniques may be used to produce an analytical stochastic variance option pricing formula.

For option pricing, much interest centers around the values of $\beta, \sigma$, and $\rho$, which determine the ways in which the distribution of $S_{t}$ departs from the lognormal. Kurtosis depends in large part on the magnitude of $\sigma$ relative to that of $\beta$. If $\sigma$ is relatively large, more "volatile" variance will lead to fat tails, raising the prices of all options far from at-the-money. Skewness depends in addition on $\rho$, and it has often been argued that the typical downward slope of Black-Scholes implied volatilities of equity options across moneyness (the "leverage" effect) is evidence of

a negative value of $\rho$. Negative return shocks are associated with positive variance shocks, making even more negative returns probable. The higher probability of such returns, relative to the lognormal, makes options with low strike prices comparatively expensive.

Unfortunately, the square root specification is generally rejected. While Andersen, Benzoni, and Lund (2000) find that the rejection is due primarily to the insufficient kurtosis generated by the model, Pan (2000) rejects the square root model based on its implications for the term structure of volatility. Other rejections are reported by Chernov and Ghysels (2000), Benzoni (1999), and less formally by Bakshi, Cao, and Chen (1997).

Subsequent generalizations to the model have included stochastic interest rates (Scott, 1997, Bakshi, Cao, and Chen, 1997), stochastic dividends (Pan, 2000), and jumps (Bates, 2000, Bakshi, Cao, and Chen, 1997, Scott, 1997, Duffie, Pan, and Singleton, 1999, and Pan, 2000). Of the three, only the inclusion of jumps has shown much promise for reversing the rejections of the model.

For several reasons, however, the econometric analysis of jumps in option pricing has proven to be problematic. First, small sample problems may be severe because jumps that 
are large enough to have a substantial effect on options prices must be infrequent in order to be consistent with the underlying returns data. These types of large jumps are seldom, if ever, found in empirical analysis of jump-diffusions applied to broad market indexes. ${ }^{2}$ Secondly, although jumps may help fit the prices of options, including them is not necessarily beneficial for the purpose of option hedging. Bakshi, Cao, and Chen (1997 and 2000) find that adding jumps to the square root stochastic variance model has little effect on pricing or hedging longer maturity options and actually worsens hedging performance for short maturities. This result is somewhat surprising since adding jumps leads to the greatest improvement in the pricing of short maturity options, and it raises the possibility that including jumps represents an overfitting of the data.

In this paper, I focus on stochastic variance alone as the source of non-Gaussian return dynamics. While including jumps will naturally improve the fit of options prices, it seems reasonable to investigate more general forms of the one-factor stochastic variance model before pursuing additional extensions such as jumps, particularly because of the problems with jumps and the soon-to-be obvious misspecification of the square root model. ${ }^{3}$

\subsection{Stochastic specification}

The goal in this section is to propose a more general parametric form of stochastic variance process than has been considered previously. The motivation is empirical, and it is based on the a casual inspection of Figure 1A, which plots the VIX index, an index of the Black-Scholes implied volatility of S\&P 100 index options, from January 1986 to April 1999. The plot is truncated at 50 percent for visual clarity; in the two weeks following the crash of October 1987, implied volatilities between 70 and 150 percent were recorded. Figure 1B plots the absolute change in the VIX index versus its level at the previous close over the same period.

From both 1A and 1B it appears that periods of high volatility coincide with periods of volatile volatility, an observation that appears to be at odds with Heston's square root formu-

\footnotetext{
${ }^{2}$ When jump processes are estimated using the time series of returns only, the jump intensity estimate tends to be high and the jump size small. Studies that find relatively small jumps include Andersen, Benzoni, and Lund (2000), Ball and Torous (1985), and Jorion (1989). Pan (2000) uses the information in options prices to help identify the jump parameters, but she is still unable to identify all the parameters of the model. To get precise estimates of the jump intensity parameter she assumes that it is the same under both the objective and risk-neutral measures, thereby sacrificing the ability to fully differentiate these measures. This makes it difficult to attribute the volatility smile to the objective probability of a market crash or investors' aversion towards such phenomena.

${ }^{3}$ Poteshman (1998) also has this goal.
} 
lation. We can see this by deriving the stochastic volatility (as opposed to variance) process implied by Heston's model.

Let $v_{t}$ denote the time $t$ instantaneous volatility, so $v_{t}=\sqrt{V_{t}}$. By Ito's lemma,

$$
d v_{t}=-\frac{\beta}{2 v_{t}}\left(-\frac{\alpha}{\beta}+\frac{\sigma^{2}}{4 \beta}-v_{t}^{2}\right) d t+\frac{1}{2} \sigma d Z_{t}^{(2)}
$$

The equation shows that the volatility of instantaneous volatility (as opposed to the volatility of instantaneous variance) is not level-dependent under the square root variance model.

Because level dependence does seem to be a feature of the data, this paper will consider a somewhat broader class of models for stochastic variance. The first generalization I consider is identical to the one offered for interest rate processes by Chan, Karolyi, Longstaff, and Sanders (1992). The square root in the variance diffusion term is simply replaced by an exponent of undetermined magnitude, so that we have

$$
\begin{aligned}
& d S_{t}=\mu S_{t} d t+\sqrt{V_{t}} S_{t} d Z_{t}^{(1)} \\
& d V_{t}=\left(\alpha+\beta V_{t}\right) d t+\sigma V_{t}^{\gamma_{1}} d Z_{t}^{(2)},
\end{aligned}
$$

where again $\operatorname{Corr}\left(d Z_{t}^{(1)}, d Z_{t}^{(2)}\right)=\rho d t$.

Following Chan et al., this constant of elasticity of variance (CEV) specification has been investigated in interest rates in a number of papers, and has been applied to stochastic variance processes by Chacko and Viceira (1999) and Lewis (2000). In empirical analysis of US short-term interest rates, starting with Chan et al., typical estimates of $\gamma_{1}$ are around 1.5. Conley, Hansen, Luttmer, and Scheinkman (1997) recently noted that such processes will have stationary distributions even when $\beta$ is positive, making mean reverting drift an unnecessary condition for stationarity.

It will be convenient to reparameterize the model to work with uncorrelated Brownian motions $B_{t}^{(1)}$ and $B_{t}^{(2)}$. Henceforth I will maintain $\operatorname{Corr}\left(d B_{t}^{(1)}, d B_{t}^{(2)}\right)=0$ and introduce correlation between $S_{t}$ and $V_{t}$ by rewriting the model as

$$
\begin{aligned}
& d S_{t}=\mu S_{t} d t+\sqrt{V_{t}} S_{t} d B_{t}^{(1)} \\
& d V_{t}=\left(\alpha+\beta V_{t}\right) d t+\sigma_{1} V_{t}^{\gamma_{1}} d B_{t}^{(1)}+\sigma_{2} V_{t}^{\gamma_{1}} d B_{t}^{(2)} .
\end{aligned}
$$

This is equivalent to the previous parameterization (2) once we set

$$
\sigma=\sqrt{\sigma_{1}^{2}+\sigma_{2}^{2}} \text { and } \rho=\frac{\sigma_{1}}{\sqrt{\sigma_{1}^{2}+\sigma_{2}^{2}}}
$$


Writing the model as in (3) suggests a second generalization of the variance process that falls outside the CEV class:

$$
d V_{t}=\left(\alpha+\beta V_{t}\right) d t+\sigma_{1} V_{t}^{\gamma_{1}} d B_{t}^{(1)}+\sigma_{2} V_{t}^{\gamma_{2}} d B_{t}^{(2)}
$$

By introducing separate power parameters on the two random shocks to instantaneous variance, the elasticity of variance is no longer constant, but depends instead on the level of the variance process. More importantly, by allowing $\gamma_{1}$ and $\gamma_{2}$ to differ the model allows the correlation of the price and variance processes to depend on the level of instantaneous variance. This correlation will be equal to

$$
\frac{\sigma_{1} V_{t}^{\gamma_{1}}}{\sqrt{\sigma_{1}^{2} V_{t}^{2 \gamma_{1}}+\sigma_{2}^{2} V_{t}^{2 \gamma_{2}}}}
$$

One can easily see that the correlation will increase in magnitude as $V_{t}$ gets larger if $\gamma_{1} / \gamma_{2}>1$. I will call this specification the 2GAM model.

As other studies have done, I impose an admittedly ad hoc form for the variance risk premium and assume that the form of the risk-neutralized process is identical to that under the true measure. Replacing the stock price drift with the instantaneous interest rate $r$ and replacing the variance drift parameters $\alpha$ and $\beta$ with their risk-neutral counterparts $\alpha^{*}$ and $\beta^{*}$, we have

$$
\begin{aligned}
& d S_{t}=r S_{t} d t+\sqrt{V_{t}} S_{t} d B_{t}^{(1) *} \\
& d V_{t}=\left(\alpha^{*}+\beta^{*} V_{t}\right) d t+\sigma_{1} V_{t}^{\gamma_{1}} d B_{t}^{(1) *}+\sigma_{2} V_{t}^{\gamma_{2}} d B_{t}^{(2) *} .
\end{aligned}
$$

Besides being arbitrary, allowing $\alpha$ and $\alpha^{*}$ to differ could potentially lead to arbitrage opportunities, since any difference between the two will cause the price of variance risk to become infinite if $V$ goes to zero. In section 4.4.1 I will show that this is unlikely to be a cause of concern for either the CEV or 2GAM model.

\subsection{Regularity}

One drawback to the variance processes in (2) and (4) is that they do not generally satisfy commonly-used sufficient conditions for a number of imporant results. The problem stems from the fact that global growth and Lipschitz conditions may be violated by the diffusion function depending on the values of $\gamma_{1}$ and $\gamma_{2}$. For many purposes (such as proving existence and uniqueness of solutions) violations of these conditions are unproblematic, but they simplify the analysis considerably. 
For the CEV process, the growth condition will be violated as $V \rightarrow \infty$ if $\gamma_{1}>1$, while the Lipschitz condition is violated as $V \rightarrow 0$ if $\gamma_{1}<1$. For the 2 GAM model, the two conditions can be violated simultaneously if $\gamma_{1}<1<\gamma_{2}$ or $\gamma_{2}<1<\gamma_{1}$.

While there are a number of ways to alter the model to impose growth and Lipschitz conditions, the above violations are most easily avoided by imposing upper and lower reflecting bounds, $V^{L}>0$ and $V^{U}<\infty$, on the volatility process. The bounds will be set low enough and high enough, respectively, so that the probability of hitting them will be extremely low and for practical purposes irrelevant.

\subsection{Expected average variance and option price approximations}

Unfortunately, the cost of the generalizations proposed is the loss of closed form option pricing formulas. While not offering closed form prices, the CEV and 2GAM models may still provide accurate approximate solutions for the prices of relatively short-term at-the-money options.

The approximation is based on Hull and White's (1987) argument that when the price and variance processes are uncorrelated, the price of an option under any stochastic variance model could be computed as the expectation of the Black-Scholes price with the variance argument replaced by the random future realization of average variance over the life of the option. If variance is not priced, as Hull and White assume, then the expectation is taken under the true measure. In general, however, if $\alpha \neq \alpha^{*}$ or $\beta \neq \beta^{*}$, then the expectation would be taken under the risk-neutral measure.

Let $\bar{V}$ denote the average variance over the life of the option, or

$$
\bar{V}_{t, t+\tau}=\frac{1}{\tau} \int_{s=t}^{t+\tau} V_{s} d s
$$

Because the Black-Scholes formula is close to linear in its variance argument for short-term at-the-money options, approximating $E^{Q}[B S(\bar{V})]$ by $B S\left(E^{Q}[\bar{V}]\right)$ may not result in large approximation errors, as the Jensen's inequality error will tend to be small.

Correlation between the price and variance process presents an additional reason to invalidate this approximation, but the practical importance of correlation is unclear for at-the-money options. To the extent that price-variance correlation and skewness are related, the analysis of Jarrow and Rudd (1982) suggests that for these options the additional approximation error caused by nonzero correlation is likely to be small. In any case, the validity of the approximation will be checked later in the paper. 
Approximating the Black-Scholes implied variance by the expected average future variance is especially attractive for stochastic variance models such as CEV or 2GAM because expected average variance can be computed easily. In fact, any process that has linear drift and that satisfies some regularity conditions (in this case guaranteed by the bounds placed on the variance process) has an expected average variance that is linear in the current state of instantaneous variance. As shown in the appendix, in this case we have

$$
\begin{gathered}
E_{t}^{Q}\left[\bar{V}_{t, t+\tau}\right]=A+B V_{t}, \\
\text { where } B=\frac{1}{\beta^{*} \tau}\left(e^{\beta^{*} \tau}-1\right) \text { and } A=-\frac{\alpha^{*}}{\beta^{*}}(1-B) .
\end{gathered}
$$

$E_{t}\left[\bar{V}_{t, t+\tau}\right]$ is therefore a weighted average of the instantaneous variance $V_{t}$ and the quantity $-\frac{\alpha^{*}}{\beta^{*}}$, the value at which the variance process has zero drift under the risk-neutral measure when $\beta^{*}<0$.

By using Black-Scholes implied variance as a proxy for the quantity on the left hand side, $E_{t}^{Q}\left[\bar{V}_{t, t+\tau}\right]$, the relation can be used to provide some information about the otherwise unobservable process $V_{t}$. Even once error is introduced into the equation, the equation can be used to draw more precise inference about the path of $V_{t}$ than would be possible using returns alone. This, in turn, will lead to more precise inference about the parameters that determine the dynamics of $V_{t}$, namely $\alpha, \beta, \sigma, \gamma_{1}, \gamma_{2}$, and $\rho$.

The approximation may be invalid for two reasons. First, the mapping from instantaneous variance to Black-Scholes implied variance could be linear, but with intercept and slope coefficients that are not determined by the formulas given in (7). Second, the true mapping may be nonlinear, invalidating the entire approach.

If neither is the case, the relation between implied variance and instantaneous variance can be used to estimate the parameters of the risk-neutral distribution, $\alpha^{*}$ and $\beta^{*}$, by "inverting" the intercept and slope of that relation according to (7). To allow, at least, for the first possible type of misspecification, this "inversion" will not rely on the functional forms in (7). Instead, I will simply estimate $A$ and $B$ directly and then find the values of $\alpha^{*}$ and $\beta^{*}$ that, along with the remainder of the parameters, approximately imply the same linear relation between implied and instantaneous variance.

Since the use of this linear relation remains another possible source of misspecification, checking the validity of this assumption will be essential. It may also be interesting because implied variance is often used as a proxy for expected average variance in the large literature 
on volatility forecasting. Investigating the validity of this approximation under the CEV and 2GAM models will have implications for this literature as well.

\section{Estimation strategy}

This paper will adopt a Bayesian perspective and will use the procedure developed in Jones (1998) to compute posterior distributions for the parameters in (4) and (6). The Bayesian framework is used primarily for reasons of efficiency and tractability. It is a likelihood-based method and should therefore make full use of the information in the sample, providing exact finitesample even for very complicated models. Although Bayesian analysis is naturally suited to situations in which the researcher has some prior information, the priors used in this analysis will be nearly uninformative, so this is of limited importance.

In this section I will describe the data, specify the econometric model, review the data augmentation procedure of Jones (1998), and propose an extension to this method that will allow the information in implied volatilities to be extracted in a simple way.

\subsection{The data}

The model will be estimated using daily S\&P 100 index returns and daily implied volatilities from the S\&P 100 options market. The time period considered extends from January 2, 1986 to June 2, 2000, a sample size of 3,538 observations. In many cases I will consider the subsample beginning on July 1, 1988, which contains 2,907 observations.

The S\&P 100 was chosen over the S\&P 500 partly because of the S\&P 100's more liquid options market during the 1980s. The more compelling reason to analyze the S\&P 100 index, however, is the availability of the Chicago Board Options Exchange Market Volatility Index (VIX). This index represents an average of eight Black-Scholes implied volatilities from options on the S\&P 100 index. The construction of the VIX index is described in detail in Whaley (1993), but the essential features are described briefly.

All eight options used in constructing the VIX are near-term and near-the-money and are very actively-traded. Each option's implied volatility is computed using a binomial tree that is adjusted for time-varying dividends, changing interest rates, and the possibility of early exercise. The S\&P 100 index level used in the calculation is perfectly contemporaneous, and to mitigate the problem that some prices underlying the index may be stale the implied volatilities 
of each call and its corresponding put are averaged. Put/call averages of implied volatilities of different moneyness (all close to at-the-money) are then interpolated to produce the at-themoney implied volatility for each maturity. This term structure of implied volatilities is then interpolated to yield a hypothetical 22-day implied volatility.

The main advantage of the VIX index is the quality of the data and the method that are used to construct it. VIX is available in real time, and even at high frequencies one does not evidence of negative autocorrelation, which one would expect if there were substantial errors in the data used to construct the index.

Use of the VIX index could be criticized on the grounds that it is a hypothetical measure, since the index does not generally coincide with the implied volatility of any particular option traded that day. Similar criticism could be aimed at term structure research that uses bootstrapped and interpolated values to construct constant maturity zero coupon yields. Partly because of these concerns, I will allow for the possibility that the VIX index makes random deviations from the implied volatility of the hypothetical option that it is designed to mimic. An assessment of the magnitude of this error will be a natural by-product of the estimation procedure.

This relaxation of the purely deterministic link between data from the underlying and options markets represents a departure from previous studies that have combined data from the two markets. In theory, given the synchronous observation of the underlying asset's price and perhaps some other market fundamentals, option prices can be inverted to yield the underlying asset's exact instantaneous volatility. ${ }^{4}$ Relaxing the strictness of this relation reflects a purely empirical perspective and is similar to the allowance of additional error terms in term structure research, where theory implies, for example, that bond yields of all maturities are deterministically linked in a single-factor term structure model. The realities of asset price data, which include sources of error such as asynchronous data and bid-ask spreads, make deterministic relations as unrealistic in options. ${ }^{5}$

Although little academic research has used the VIX index, studies by Fleming, Ostdiek, and Whaley (1995) and Blair, Poon, and Taylor (1999) have found it to be a reliable indicator of future stock market volatility. Both papers find VIX to outperform measures of volatility based

\footnotetext{
${ }^{4}$ This was proved formally by Bergman, Grundy, and Wiener (1996).

${ }^{5}$ Christensen and Prabhala (1998) have argued recently that these types of measurement errors are severe enough to bias tests of the informational content of implied volatility. Additional sources of error could arise from simplifications made about the dividend process (Harvey and Whaley, 1991) or errors in the market's inference about the current state of market volatility.
} 
on past returns, with the latter paper concluding this is the case even when the returns-based measure is constructed from five-minute index returns.

\subsection{The econometric model of implied volatility}

The full model to be estimated is described by the SDEs that govern the dynamics of $S_{t}$ and $V_{t}$ under the objective measure, given by (2) or (4), and a regression-type equation that links the unobservable $V_{t}$ to an observed implied variance,

$$
I V_{t}=A+B V_{t}+\epsilon_{t}
$$

Equation (8) is simply a restatement of (7) with the addition of an error term and the replacement

of $E_{t}^{Q}\left[\bar{V}_{t, t+\tau}\right]$ by its proxy, $I V_{t}$. The time series $I V_{t}$ is a simple transformation of the VIX index: it is converted to decimals, squared to change it from a volatility to a variance, then divided by 264 to convert to a daily basis. Since the VIX index reflects a 22-day implied volatility, I set $\tau=22$.

Because of the apparent heteroskedasticity in $I V_{t}$ demonstrated in figures $1 \mathrm{~A}$ and $1 \mathrm{~B}$, the regression error $\epsilon_{t}$ will be assumed be heteroskedastic as well, with a standard deviation proportional to $I V_{t-1}$. We therefore have $\epsilon_{t} \sim$ i.i.d. $N\left(0, \xi^{2} I V_{t-1}^{2}\right)$, with $\xi$ a parameter to be estimated. The magnitude of $\xi$ will reflect the accuracy of the proxy of $E^{Q}[\bar{V}]$ by $I V$.

Allowing this form of heteroskedasticity should mitigate the concern that the level effect in the volatility of volatility apparent in Figure 1 is solely due to measurement error. If this is the case, then we may still find low values for $\gamma_{1}$ and $\gamma_{2}$.

\subsection{Computing posteriors by data augmentation}

Estimation of these models using standard methods is difficult for several reasons. First, the dynamics of $S_{t}$ and $V_{t}$ are represented as a diffusion process, making standard maximum likelihood or moment-based approaches impractical. The latency of stochastic variance further limits the effectiveness of these approaches. Lastly, the method used must simultaneously estimate the parameters of the SDE (3) or (4) and the regression equation (8).

The approach taken uses the data augmentation procedure of Jones (1998). The method is based on the idea that the Euler approximation, a discrete time Gaussian model, can be used to approximate the transition density of a diffusion process. The Euler approximation has been used in many papers, and its use in likelihood-based inference has been justified by Pedersen 
(1995) and Brandt and Santa-Clara (1999). Under regularity conditions, the Euler approximation converges to the diffusion as the time step of the Euler process shrinks to zero.

It is sufficient for convergence that growth and Lipschitz conditions are satisfied, both of which the unconstrained model may violate. While these conditions may be overly stringent, by bounding the process above and below both conditions are satisfied globally. As upper and lower bounds, I choose the values $V^{U}=1$ and $V^{L}=10^{-8}$, corresponding to daily return volatilities of 100 percent and .01 percent.

The Euler approximation for the 2GAM stochastic variance model is

$$
\begin{aligned}
& S_{(k+1) h}=S_{k h}+h \mu S_{k h}+\sqrt{h} \sqrt{V_{k h}} S_{k h} \eta_{k+1}^{(1)} \\
& V_{(k+1) h}=V_{k h}+h\left(\alpha+\beta V_{k h}\right)+\sqrt{h} \sigma_{1} V_{k h}^{\gamma_{1}} \eta_{k+1}^{(1)}+\sqrt{h} \sigma_{2} V_{k h}^{\gamma_{2}} \eta_{k+1}^{(2)},
\end{aligned}
$$

where $\eta_{k}^{(1)}$ and $\eta_{k}^{(2)}$ are independent standard normals and $k=0, \ldots, K$. Note that $S_{t}=S_{k h}$ for $k=\frac{t}{h}$.

In the estimation of the model, the stock price process $S_{t}$ will be observed at the end of each trading day. Without loss of generality, assume that these end-of-day times correspond to $t \in\{0,1,2, \ldots, T\}$. A discrete time stochastic variance model may therefore be formulated by setting $h=1$. One can see, however, that the properties of the process with $h=1$ may be far different from the same process with $h$ set much smaller. When $h=1$, the conditional distribution of $S_{t+1}$ given $S_{t}$ and $V_{t}$ is Gaussian, while for smaller $h$ it is not. One reason this distribution will deviate from normality is that $\sigma_{1} \neq 0$, implying that the stock and variance processes are correlated. If, for example, $\sigma_{1}<0$, then this correlation is negative, making a negative shock to returns likely to coincide with a positive shock to variance. This, in turn, makes larger shocks more likely and increases the probability of an even larger negative return. If $h$ is small, this feedback effect can occur inside of a single period, and the result is that the conditional distribution of $S_{t+1}$ will exhibit negative skewness. For similar reasons $S_{t+1}$ will also display excess kurtosis.

For convergence of the Euler approximation and for the generation of nonnormality in conditional one-period returns, it is therefore imperative to allow $h$ to be small in the estimation process, even if this means that the Euler approximation interval $h$ is considerably smaller than the time interval at which the data are observed. This creates a problem for likelihood and moment-based inference, since neither the moments nor the transition densities of the Euler approximation are known over intervals larger than $h$. The strategy proposed by Pedersen (1995) and Brandt and Santa-Clara (1999) is to compute the likelihoods by simulation. In 
moment-based techniques such as Gallant and Tauchen's (1996) efficient method of moments, moments are computed by simulating long paths of the Euler approximation.

Since estimation of (9) essentially amounts to the analysis of a conditionally Gaussian model with a large number of missing data points, this paper will make use of Tanner and Wong's (1987) data augmentation algorithm in a manner similar to the discrete time stochastic volatility analysis of Jacquier, Polson, and Rossi (1994). Since posterior distributions that are impossible to compute given the original data are often easily analyzed under the completed data set, data augmentation is often a useful tool for the analysis of models with latent variables.

Although we do not see the augmented data, we often know their distribution conditional on the model parameters and the data that are observed. Data augmentation expands the set of model "parameters" to include all augmented data in addition to the structural parameters (e.g. $\alpha$ and $\beta$ ). As in all Bayesian analysis, this parameter vector is treated as a random quantity, with the explicit goal being the characterization of its posterior distribution. This expanded parameter vector is quite large in the case of stochastic variance diffusions, corresponding to the set of structural parameters, values of the stock price $S_{t}$ that are realized in between the endof-day observations (all non-integer $t$ ), and the complete time series of unobserved variances $V_{t}$. Since posteriors of such high dimensioned random variables can seldom be derived analytically, numerical methods are often needed. Analysis of the models therefore proceeds using a set of tools collectively known as Markov chain Monte Carlo (MCMC). Chib and Greenberg (1996) provide an introduction to these methods.

In short, using MCMC methods we generate many random draws from the posterior distribution rather than deriving the distribution analytically. Given enough draws from the posterior, sample moments, quantiles, and even densities are easy to calculate It turns out that it is often easy to compute posteriors using MCMC when it is impossible to do so analytically.

The sequence of draws made from the posterior distribution are not serially independent, but rather form a Markov chain. Under extremely mild conditions, the chain converges in distribution to the posterior distribution. In practice, one starts the chain with an arbitrary initialization. A long chain is simulated, and the first portion of it is discarded to negate the effect of the initial values chosen. The chain is ergodic, so the remaining portion, although possibly autocorrelated, can be used to estimate posterior moments.

The two most popular forms of MCMC are the Gibbs sampler and the Metropolis-Hastings algorithm. The Gibbs sampler is a tool for drawing high-dimensional random variables by 
separating the draw into a number of smaller, more tractable draws. The Metropolis-Hastings algorithm is used to draw from a density that is known only up to a constant of proportionality. Versions of both will be used in this paper. ${ }^{6}$

The Gibbs sampler is useful in the present situation because the size of the expanded parameter vector is very large. Rather than analyzing the vector as a whole, I break it down into many small pieces, drawing each piece conditional on all the previous draws of all the other pieces. Eventually, the Markov chain formed by this procedure converges to the true posterior distribution.

More formally, suppose $\theta$ is a multidimensional random vector that can be partitioned into $I$ subvectors $\left\{\theta^{(1)}, \theta^{(2)}, \theta^{(3)}, \ldots, \theta^{(I)}\right\}$ of possibly different sizes, and suppose the goal is to generate a sequence of random draws from the distribution $p(\theta \mid X)$, where $X$ represents some data set. Choose some arbitrary initial value $\theta_{0}$ and then iteratively form a chain of $\theta_{n}=\left\{\theta_{n}^{(1)}, \theta_{n}^{(2)}, \theta_{n}^{(3)}, \ldots, \theta_{n}^{(I)}\right\}$. For $i$ between 1 and $I$, let $\theta_{n}^{(i)}$ be a random draw of $\theta^{(i)}$ from its conditional distribution $p\left(\theta^{(i)} \mid \theta_{n}^{(-i)}, X\right)$, where

$$
\theta_{n}^{(-i)}=\left\{\theta_{n}^{(j)}, j<i\right\} \bigcup\left\{\theta_{n-1}^{(j)}, j>i\right\}
$$

The result of the Gibbs sampler is that under extremely mild conditions the Markov chain $\theta_{n}$ converges in distribution to $p(\theta \mid X)$.

For the stochastic variance process, the partitions $\theta^{(i)}$ will be defined as follows:

$$
\begin{aligned}
& \cdot \theta^{(1)}=\{\mu, \alpha, \beta\} \\
& \cdot \theta^{(2)}=\left\{\sigma_{1}, \sigma_{2}, \gamma_{1}, \gamma_{2}\right\} \\
& \cdot \theta^{(3)}=\{A, B\} \\
& \cdot \theta^{(4)}=\{\xi\} \\
& \cdot\left\{\theta^{(5)}, \ldots, \theta^{T+5}\right\}=\left\{V_{t}, t=0,1, \ldots, T\right\} \\
& \cdot\left\{\theta^{(T+6)}, \ldots, \theta^{I}\right\}=\left\{\left(S_{t}, V_{t}\right), t \notin \text { integers }\right\}
\end{aligned}
$$

The data set $X$ corresponds to the observations of the stock price $S_{t}$ and the implied variance index $I V_{t}$ for $t=0,1, \ldots, T$.

\footnotetext{
${ }^{6}$ An highly readable introduction to the Gibbs sampler can be found in Casella and George (1992), while Chib and Greenberg (1995) provide an equally valuable reference for the Metropolis-Hastings algorithm.
} 
One can think about the procedure as "cycling" through the partitions $\theta^{(i)}$. Each cycle (n) begins with a draw of $\theta^{(1)}$, followed by draws of $\theta^{(2)}, \theta^{(3)}$, and $\theta^{(4)}$. The set of all end-of-day values of $V_{t}$ are cycled through next. Lastly, the algorithm cycles through the pairs of $\left(S_{t}, V_{t}\right)$ that are realized at times between the end-of-days. ${ }^{7}$

As opposed to the simulated maximum likelihood methods of Pedersen (1995) and Brandt and Santa-Clara (1999), which simulate the Euler approximation forward, the draws of high frequency data here merely "bridge" the observed low frequency data with short paths of high frequency data. Figures $2 \mathrm{~A}$ and $2 \mathrm{~B}$ illustrate the difference between the two approaches. The advantage of the latter method is that the variance of the simulated data is smaller, since it must be consistent with both the start and end points. Since both methods must effectively integrate out the dependence on particular paths of simulated data, the smaller variance makes this integration less computationally demanding. This is particularly apparent when considering latent variable models, such as stochastic volatility, where simulated maximum likelihood is generally infeasible.

The sole point of data augmentation in the time dimension is bias reduction. Data augmentation merely makes the discrete time Euler approximation less misspecified, so that the posteriors computed approach those of the true diffusion. Augmenting the price process with the variance process is simply a convenient way to approach generating parameter posteriors, as shown by of Jacquier, Polson, and Rossi (1994).

The methods for performing the draws of the structural parameters $\left(\theta^{(1)}\right.$ to $\left.\theta^{(4)}\right)$ are contained in the appendix, but the most important details can be described briefly. In drawing these parameters, virtually "flat" priors are used exclusively for all parameters except $\xi$, for which a small amount of prior information was found to be sometimes necessary. Each parameter is assumed independent of the others in the prior, with the joint prior given by

$$
p\left(\mu, \alpha, \beta, \sigma_{1}, \sigma_{2}, \gamma_{1}, \gamma_{2}, \alpha^{*}, \beta^{*}, \xi\right) \propto \frac{1}{\sigma_{2}} f(\xi),
$$

where $f(\xi)$ is the inverted gamma distribution with 15 degrees of freedom and gamma parameter $\frac{40}{3} .8$ The posterior distribution is computed to be proportional to the prior times the complete likelihood, which includes both the product of transition densities and the likelihood

\footnotetext{
7 Although the number of partitions $I=\frac{T}{h}+6$ can be very large, often close to half of the $I$ draws can be performed simultaneously, reducing computing time considerably. Because the Euler approximation is a Markov process, only adjacent observations $(t-h$ and $t+h)$ are relevant for each draw of $V_{t}$ or $\left(S_{t}, V_{t}\right)$. We can therefore draw every other $\theta^{(i)}$ (for $i>4$ ) simultaneously and alternate between just a few matrix draws rather than $I$ individual draws. Describing the method as "cycling" through the $I$ draws is for expositional convenience only.

${ }^{8}$ This inverted gamma distribution would result as the posterior distribution following the observation of 15
} 
of the initial observation $\left(S_{0}, V_{0}\right)$. Since $S_{0}$ is nonstationary, its density is assumed to be diffuse, and so the joint density of $\left(S_{0}, V_{0}\right)$ is replaced with the unconditional density of just $V_{0}$.

\subsection{Drawing the augmented data}

Let $\theta^{S}$ denote the collection of structural parameters, $\theta^{(1)}$ to $\theta^{(4)}$, let $\mathbf{S}_{-t}$ and $\mathbf{V}_{-t}$ denote the unions of all $S_{s}$ and $V_{s}$ for $s \neq t$, and let IV denote the entire time series of $I V_{t}$. In this section I will describe the methods for drawing the latent realizations of $S_{t}$ and $V_{t}$ given $\theta^{S}, \mathbf{S}_{-t}, \mathbf{V}_{-t}$, and IV.

Noninteger $t$ correspond to times other than the end-of-days, so neither $S_{t}$ nor $V_{t}$ are observed for these $t$. Since we observe implied variance contemporaneously with the stock price, $I V_{t}$ is also unobserved. Our goal for these $t$ is therefore to draw $S_{t}$ and $V_{t}$ from

$$
p\left(S_{t}, V_{t} \mid \theta^{S}, \mathbf{S}_{-t}, \mathbf{V}_{-t}, \mathbf{I V}\right)
$$

Since we are already conditioning on $\mathbf{V}_{-t}$, the additional conditioning on IV is irrelevant, since past and future values of $I V$ just provide noisy signals about the contemporaneous values of $V$. The Markovian nature of the model further makes observations of $S$ and $V$ at times before $t-h$ and after $t+h$ irrelevant as well. The draw therefore reduces to the somewhat simpler

$$
p\left(S_{t}, V_{t} \mid \theta^{S}, S_{t-h}, V_{t-h}, S_{t+h}, V_{t+h}\right) .
$$

Invoking Bayes rule, this can be shown to be proportional to

$$
p\left(S_{t}, V_{t} \mid \theta^{S}, S_{t-h}, V_{t-h}\right) p\left(S_{t+h}, V_{t+h} \mid \theta^{S}, S_{t}, V_{t}\right) .
$$

For shorthand that will be useful below, let

$$
\pi\left(S_{t}, V_{t}\right)=p\left(S_{t+h}, V_{t+h} \mid \theta^{S}, S_{t}, V_{t}\right) .
$$

The importance of (11) is that the first density is a bivariate Gaussian density for $\left(S_{t}, V_{t}\right)$ and can therefore can be simulated. The second density is Gaussian as well, but for $\left(S_{t+h}, V_{t+h}\right)$, not $\left(S_{t}, V_{t}\right)$, and it will generally not be the case that the product of the two densities is a standard density for $\left(S_{t}, V_{t}\right)$. For this reason, and also because the constant of integration is unknown, I use the Metropolis-Hastings algorithm to draw $\left(S_{t}, V_{t}\right)$ from the product of these densities.

data points with a sample standard deviation of .1 with the diffuse prior $p(\xi) \propto \frac{1}{\xi}$. The sample sizes used in the analysis are all over 2,500, making $f(\xi)$ a relatively uninformative prior. With a truly diffuse prior, however, the algorithm occasionally approached a fixed point at $\xi=0$. 
Specifically, I will draw a candidate pair $\left(S_{t}^{*}, V_{t}^{*}\right)$ according to $p\left(S_{t}, V_{t} \mid \theta^{S}, S_{t-h}, V_{t-h}\right)$ and use it to replace the previous draw $\left(S_{t}, V_{t}\right)$ with probability

$$
\min \left\{\frac{\pi\left(S_{t}^{*}, V_{t}^{*}\right)}{\pi\left(S_{t}, V_{t}\right)}, 1\right\} \text {. }
$$

Intuitively, the values drawn are those that would likely follow $\left(S_{t-h}, V_{t-h}\right)$. Out of these draws, the ones accepted are those that are likely to precede $\left(S_{t+h}, V_{t+h}\right)$. The MetropolisHastings result is that the Markov chain constructed from such draws will converge to a distribution whose density is proportional to the product of the two densities in (11).

The second main type of draw is for integer $t$, where $S_{t}$ is observed but $V_{t}$ is not. A contemporaneous value of implied variance $I V_{t}$ is observed as well. Slightly different from before, we would like to draw from

$$
p\left(V_{t} \mid S_{t}, \theta^{S}, \mathbf{S}_{-t}, \mathbf{V}_{-t}, \mathbf{I V}\right)
$$

In this case, the implied variance time series is informative, because $I V_{t}$ provides information about $V_{t}$, and $V_{t}$ is not contained in $\mathbf{V}_{-t}$. As before, however, past and future values of $I V$ are irrelevant given past and future values of $V$. In addition, the Markovian nature of the model again makes observations of $S$ and $V$ at times before $t-h$ and after $t+h$ irrelevant. The density therefore simplifies to

$$
p\left(V_{t} \mid \theta^{S}, S_{t-h}, V_{t-h}, S_{t}, S_{t+h}, V_{t+h}, I V_{t}\right)
$$

which by Bayes rule is proportional to

$$
p\left(V_{t} \mid \theta^{S}, S_{t-h}, V_{t-h}, S_{t}, I V_{t}\right) p\left(S_{t+h}, V_{t+h} \mid \theta^{S}, S_{t}, V_{t}\right)
$$

While the second density is the same as before, the first is different because of two additional conditioning arguments, $S_{t}$ and $I V_{t}$. Again invoking Bayes rule, we may further decompose the first density and show

$$
p\left(V_{t} \mid \theta^{S}, S_{t-h}, V_{t-h}, S_{t}, I V_{t}\right) \propto p\left(V_{t} \mid \theta^{S}, S_{t-h}, V_{t-h}, S_{t}\right) p\left(I V_{t} \mid \theta^{S}, S_{t-h}, V_{t-h}, S_{t}, V_{t}\right)
$$

Eliminating irrelevant conditioning arguments, we have

$$
p\left(V_{t} \mid \theta^{S}, S_{t-h}, V_{t-h}, S_{t}, I V_{t}\right) \propto p\left(V_{t} \mid \theta^{S}, S_{t-h}, V_{t-h}, S_{t}\right) p\left(I V_{t} \mid \theta^{S}, V_{t}\right) .
$$

The first density is now the same as before, and the second reflects the information contribution of the implied variance series. Both densities are Gaussian, so the product of the two is proportional to a Gaussian density as well (with mean and variance given in the appendix). 
The Metropolis-Hastings candidate $V_{t}^{*}$ is now generated according to the distribution

$$
p\left(V_{t} \mid \theta^{S}, S_{t-h}, V_{t-h}, S_{t}, I V_{t}\right) .
$$

The acceptance probability is identical to the previous one, except that $S_{t}$ now replaces $S_{t}^{*}$. In practice, the acceptance rates of both Metropolis draws are typically above 50 percent.

Draws of the first and last observations of $V-V_{0}$ and $V_{T}$ - require slight modifications of the procedure. For $V_{T}$, the candidate is generated as before, but $T+h$ term disappears, making the acceptance probability equal to one. For $V_{0}$, the density $p\left(V_{t} \mid \theta^{S}, S_{t-h}, V_{t-h}, S_{t}\right)$ is replaced by the unconditional density $p\left(V_{0} \mid \theta^{S}\right)$. It will be shown later that this density can usually be computed up to a constant of proportionality, although it is of an unknown form. Hence the candidate is generated only using the density $p\left(I V_{0} \mid \theta^{S}, V_{0}\right)$, with the unconditional density relegated to the acceptance probability, which becomes

$$
\min \left\{\frac{\pi\left(S_{0}, V_{0}^{*}\right) p\left(I V_{0} \mid \theta^{S}, V_{0}^{*}\right)}{\pi\left(S_{0}, V_{0}\right) p\left(I V_{0} \mid \theta^{S}, V_{0}\right)}, 1\right\} .
$$

Because the augmented variance process is drawn conditional on the observed data, it will only take on values that are consistent with the returns and implied variances that were observed in the sample. This almost certainly eliminates the possibility of volatilities hitting the lower bound of .01 percent or the upper bound of 100 percent, since there were no sample returns or implied variances that suggested that actual market volatility was ever close to either of these extreme levels. For this reason I ignore the reflecting bounds in the estimation of the model, and I am confident that they would not be hit were they imposed. Since the bounds may be more important in simulating purely hypothetical samples of data, however, they will be imposed in all post-estimation analysis.

\section{Results}

Three versions of the model will be considered. The unrestricted version is the 2GAM model as it was described in (4). The first restricted version is the CEV model, in which the constraint $\gamma_{1}=\gamma_{2}$ is imposed. The final version (the SQRT model) imposes the additional restriction that

$\gamma_{1}=\frac{1}{2}$, and is therefore identical to Heston's square root (1993) model. Both versions will be estimated over the full sample of January 1986 to June 2000 and the post-crash sample, which begins in July 1988. Six hundred thousand iterations of the estimation algorithm are made, with the first hundred thousand discarded to eliminate dependence on the initial conditions. 
In this section I will begin with a summary of the posterior distributions of the model parameters, followed by an assessment of the validity of the implied variance proxy used to compute these posteriors. The focus will then shift to the implications of the results for the distribution of returns, and I will examine both the higher moments and the options prices generated by each model.

\subsection{Parameter posteriors}

Table 1 presents summary statistics on the posterior distributions of the model parameters computed using $h=\frac{1}{10} .9$

The first two columns of the table contain posterior means and standard deviations of the parameters of the restricted (SQRT) model. The second two columns report the same statistics for the CEV model, and the last two contain results for the 2GAM model. I report posterior statistics for the ten parameters of the model as well as two simple transformations of these parameters The first transformation, $\rho(.0001)$, is the correlation between the price and variance processes when $V=.0001$, computed from (5). This value of $V$ corresponds to a daily volatility of one percent, a fairly typical day for the S\&P 100 index. (For the CEV and SQRT models, this correlation does not depend on $V$.) Second, I report $\sigma(.0001)$, the volatility of instantaneous variance given $V=.0001$.

For the SQRT model, results vary appreciably across the two samples. This attests to the large effect the crash of October 1987 has on the estimates, since the second sample only differs from the first in its exclusion of the months prior to July 1988. One difference is that the variance persistence parameter $\beta$ is much lower under the full sample, implying a variance process that is apparently much faster to return to its long run mean. This is consistent with Schwert's (1990a) observation that volatility returned to normal levels especially quickly following the crash of ' 87.

The parameters $A$ and $B$ that govern the relation between implied and instantaneous variance are comparatively stable across sample periods for the SQRT model, suggesting that on average this relation was stable during the 1987 crash. However, there appears to be a great deal of noise in this relation during the crash period. The parameter $\xi$, which provides an

\footnotetext{
${ }^{9}$ Similar results are obtained by setting $h=\frac{1}{3}$, but results are noticeably different from those obtained using $h=1$, suggesting that discretization bias is potentially a problem but that a relatively small amount of augmented data leads to the approximate convergence of posteriors.
} 
indication of how well the instantaneous variances of each model fit the time series of implied variances, is much larger when estimated over the whole sample.

The SQRT posterior differs in several other ways across the two sample periods. First, the correlation of the price and variance processes is around -.4 in the whole sample, but increases in magnitude to -.7 for the subsample. Since this correlation is the primary determinant of skewness in returns, these numbers have very different implications for the volatility smile. Second, on a typical day with instantaneous daily standard deviation of one percent, the volatility of instantaneous variance, $\sigma(.0001)$, is roughly twice as high for the whole sample than it is for the subsample, which should generate much fatter tails and also alter the implied volatility smile.

The first main empirical finding of the paper concerns the CEV model and is found in the two middle columns of Table 1. In both the whole sample and the post-July 1988 subsample, I find precise estimates of $\gamma_{1}$ that are greater than one, capturing the level dependence of the volatility of implied volatility that was evident in Figure 1. The correlation of the price and variance processes is even larger in magnitude under the CEV model, with a value of -.86 over the entire sample and -.73 in the post-crash subsample. The volatility of instantaneous variance on a typical day, $\sigma(.0001)$, is lower than it is for the SQRT model and is somewhat closer across the two sample periods.

The second main result is that the additional generalization to the $2 \mathrm{GAM}$ model appears to be important. The last columns of Table 1 show that the $\gamma_{1}$ parameter is substantially higher than $\gamma_{2}$ for both sample periods. Since $\sigma_{1}<0$, this will have the effect of making the correlation between the price and variance processes more negative as volatility increases. In many other aspects, the CEV and 2GAM models are quite similar, with drift parameters that are extremely close to zero over the 1988-2000 sample.

As in the SQRT model, under the CEV and 2GAM models $\alpha$ and $\beta$ differ significantly across the two samples, although for both models the drift of the variance process looks less mean reverting when the crash period is included in the sample, a surprising result given Schwert's (1990a) observation. The intercept and slope parameters $A$ and $B$ are also very different across samples, suggesting a more serious parameter constancy problem for risk premia estimates.

One potential concern is that the posterior mean of $\beta$ is positive for the CEV and 2GAM models over the entire sample period. Over the shorter 1988-2000 sample, much of the posterior mass of $\beta$ lies above zero for both the CEV and 2GAM models, although the posterior 
means are both negative. These results might lead one to conclude that the variance processes is explosive. Conley, Hansen, Luttmer, and Scheinkman (1997) show, however, that this conclusion is false, because stationarity will be generated through the diffusion term as long as either $\gamma_{1}$ or $\gamma_{2}$ are greater than one. Below I will examine these stationary densities directly.

The parameter $\xi$, which measures the goodness of fit of the option implied variances, decreases once the crash period is excluded, as it did for the SQRT model. For the SQRT model, however, this improvement is dramatic, while for the CEV and 2GAM models it is somewhat more modest. This suggests that the SQRT model is incapable of explaining crash volatilities, even in-sample, while the CEV and 2GAM models do comparatively well. For the post-July 1988 period, the CEV and 2GAM models provide a more moderate improvement.

Overall, all models display parameter constancy problems when the 1986-1988 crash period is removed from the sample, consistent with the apparent regime shift in index option pricing noted by Bates (2000). For the SQRT model, the largest differences were found in the correlation of the price and variance processes and the volatility of instantaneous variance. For the CEV model, these numbers were somewhat closer across the two samples, as was the CEV parameter, $\gamma_{1}$, and similar results are found for the 2GAM model. Changes in the drift parameters $\alpha$ and $\beta$ were not quite as severe for the CEV and 2GAM models, although the changes in the regression parameters $A$ and $B$ are more noticeable for CEV and 2GAM. In short, the results for the SQRT model imply that the true returns process changed following the crash of 1987, while the CEV and 2GAM model results suggest that it is primarily the pricing of volatility risk that changed since then.

Finally, the high variance elasticity found here it shared by the the process estimated by Poteshman (1998) using a nonparametric approach. He also finds a diffusion function that is convex, at least for low to moderate levels of $V$. The level dependence of the volatility of volatility that is implied by the CEV model is also similar to the so-called "GARCH diffusion" model, in which $\gamma_{1}=\gamma_{2}=1$, and to the EGARCH models proposed by Nelson (1991) and favored by Pagan and Schwert (1990). It is, in addition, a characteristic of the GARCH specification estimated by Hentschel (1995).

Although a direct comparison of these latter discrete-time models with the continuous-time models considered in this paper is somewhat difficult, we can gain some intuition by contrasting the CEV specification with a continuous-time model of the logarithm of instantaneous variance We may view the Ornstein-Uhlenbeck process for log variance as an approximation of a simple 
EGARCH model:

$$
d \log V_{t}=\kappa\left(\theta-\log V_{t}\right) d t+\sigma d B_{t}
$$

Applying Ito's lemma, the following process for $V_{t}$ may be derived:

$$
d V_{t}=V_{t} \kappa\left(\theta+\frac{\sigma^{2}}{2 \kappa}-\log V_{t}\right) d t+\sigma V_{t} d B_{t}
$$

Like the CEV process, the volatility of this variance process is also highly dependent on the level of instantaneous variance, although the slightly convex CEV diffusion function I estimate should lead to a variance process capable of reaching somewhat higher levels. The nonlinear drift of the new variance process will also tend to keep variance from growing too large, as the strength of mean reversion now increases with the level of instantaneous variance. Combined, these differences should cause the CEV model to exhibit substantially greater non-normality in returns.

\subsection{Checking the implied variance approximation}

The previous results were dependent on the assumption that the implied variance of a 22-day at-the-money option was an unbiased, if noisy, proxy for the expected average variance under the risk-neutral distribution over the life of that option. This assumption was used to motivate a linear relation between the instantaneous variance $V_{t}$ and the implied variance $I V_{t}$.

Since this linear relation was derived as an approximation, it is important to verify that it holds reasonably well. In this section, I assess the validity of the linearity assumption under the parameter estimates reported in Table 1. Most of the remainder of the paper will focus on estimates from the post-July 1988 subsample, so the results reported in this section will be based on the posterior means for the 1988-2000 sample period. In addition, to alleviate concerns that the approximation might fail for other parameter values, causing the posterior distributions to be distorted away from these values, I check the same approximations over a more "challenging" range of parameter vectors.

Given a vector of parameters $\left(\alpha, \beta, \sigma_{1}, \sigma_{2}, \gamma_{1}, \gamma_{2}\right)$ and a level of instantaneous variance, I compute the Black-Scholes implied variance corresponding to a hypothetical 22-day at-themoney option. ${ }^{10}$ Figures $3 \mathrm{~A}$ to $3 \mathrm{D}$ show the relation between $V_{t}$ and $I V_{t}$ for the SQRT model over the range $V=.00001$ to .001, which corresponds to annualized volatilities between $5 \%$

\footnotetext{
10 Generation of these implied variances requires the calculation of option prices, which are computed by simulation in a manner described later in the discussion of option pricing.
} 
and 50\%. The top left panel (3A) displays the relation for the estimated posterior means, and almost perfect linearity is apparent.

Since the Jensen's inequality error in the linearity approximation is likely worsened when the variance of $V$ increases, Figure 3B shows the same relation where both $\sigma_{1}$ and $\sigma_{2}$ are increased by a factor of $\sqrt{1.5}$, corresponding to a $50 \%$ increase in the instantaneous variance of $V$. In Figure 3C, the degree of model nonlinearity is increased by raising $\gamma_{1}$ by 25\%, to 625 . In addition, $\sigma_{1}$ and $\sigma_{2}$ are also increased by factor sufficient to keep the overall variance of $V$ comparable to that implied by the original estimates. Finally, in Figure 3D the degree of negative correlation between returns and volatility is strengthened by increasing $\sigma_{1}^{2}$ by $50 \%$ and reducing $\sigma_{2}^{2}$ by a third. In all cases, the linear relation in Figure 3A is virtually unchanged, suggesting that the linear approximation of $I V_{t}$ is unlikely to be a source of error for the SQRT model.

Figures $4 \mathrm{~A}$ to $4 \mathrm{D}$ repeat the same set of experiments for the CEV specification. As before, the $V_{t} / I V_{t}$ relation is approximately linear under the estimated parameter means. The approximation appears to remain reasonable in Figure $4 \mathrm{~B}$, when the variance of the $V_{t}$ process is raised, and Figure 4D shows that increasing the price/variance correlation also has little impact. In Figure $4 \mathrm{C}$, however, a slight nonlinearity is detectable as $\gamma_{1}$ is increased from 1.22 to 1.53. The relation between $V_{t}$ and $I V_{t}$ now appears concave, raising the possibility that the posterior statistics reported in Table 1 for the CEV model are inaccurate. Specifically, the parameter vectors in the reported posterior distribution could be skewed towards values for which the approximation holds, namely lower values of $\gamma_{1}$. Because of the large distance between 1.53 and the posterior distribution reported for $\gamma_{1}$, it is unlikely that the impact of any bias is large. If a bias does exist, it is likely that $\gamma_{1}$ is biased downward, which would preserve the paper's finding that $\gamma_{1}$ is greater than one.

Results for the 2GAM model are presented in Figures 5A to 5D. Nonlinearity, although slight, is now observed in Figure 5A for the estimated parameter vector. The problem appears to be more severe using the three other parameter vectors. As before, the concern is that the computed posterior distribution is tilted away from parameter vectors for which the approximation does not hold. If this is the case, then higher values of $\sigma_{1}, \sigma_{2}, \gamma_{1}$, and $\gamma_{2}$ would all be more likely than those reported in Table 1, and the price/volatility correlation would likely be stronger as well. 


\subsection{The risk-neutral drift}

In addition to providing information about the time series of $V_{t}$, the relation between $V_{t}$ and $I V_{t}$ can be used to extract estimates of the risk-neutral parameters $\alpha^{*}$ and $\beta^{*}$, since they determine the intercept and slope the approximate linear relation, as motivated by (7).

Even if the formulas in (7) do not hold, there may still exist an approximately linear relation between $I V_{t}$ and $V_{t}$. In this section, I infer $\alpha^{*}$ and $\beta^{*}$ from the estimates of $A, B$, and the other parameters. To do so I will use the posterior means from Table 1, along with some values $\alpha^{*}$ and $\beta^{*}$, to determine the true model-implied relation between $I V_{t}$ and $V_{t}$. Estimates of $\alpha^{*}$ and $\beta^{*}$ will be those values that minimize the difference between the true and approximate relations.

More formally, let $V_{i}, i=1, \ldots, 25$, denote 25 equally-spaced levels of instantaneous variance, corresponding to daily volatility levels between $1 \%$ and $25 \%$. Let $\hat{A}$ and $\hat{B}$ denote estimates of $A$ and $B$ and let $\hat{\theta}$ denote a vector of the remaining parameter estimates, $\left(\hat{\alpha}, \hat{\beta}, \hat{\sigma}_{1}, \hat{\sigma}_{2}, \hat{\gamma}_{1}, \hat{\gamma}_{2}\right)$. Let $I \hat{V}_{i}$ denote the Black-Scholes implied variances that are generated by the true model under the parameter values $\hat{\theta}$. The estimates of $\alpha^{*}$ and $\beta^{*}$ are the values that minimize the sum of squared errors measure

$$
\sum_{i=1}^{25}\left(I \hat{V}_{i}-\hat{A}-\hat{B} V_{i}\right)
$$

As before, I focus on estimates from the post-July 1988 subsample, so the results reported in this section will set $\hat{\theta}$ equal to the posterior means calculated over this period. In principle, one could calculate a pseudo-posterior distribution of $\alpha^{*}$ and $\beta^{*}$ by recomputing these values for each draw of the model parameters. Since the SSE minimization is a highly numerical procedure, I have not done so, making precise inferences about $\alpha^{*}$ and $\beta^{*}$ difficult.

The results of the minimization are reported in Table 2. In all three cases, the estimates of $\beta^{*}$ are positive. For the SQRT model, this implies that the variance process is explosive under the risk-neutral measure, a deficiency of the SQRT model that has been noted by Pan (2000), among others. For the CEV model and 2GAM models, the estimates of $\beta^{*}$ are even larger, which would imply highly explosive variance processes were the diffusion function a constant. For reasons discussed in the next section, the CEV and 2GAM processes are actually stationary despite their drift, since their stationarity is instead volatility-induced. 


\subsection{Stationary and dynamic behavior of $\mathrm{V}$}

\subsubsection{Stationary distributions}

The posterior mean values reported in Table 1 all generate stationary processes under both the objective measure. For the SQRT model, stationarity is achieved through mean reversion, since $\beta<0$ for both sample periods. For the CEV and 2GAM model, the high values of $\gamma_{1}$ and $\gamma_{1}$ make the signs of $\beta$ and $\beta^{*}$ irrelevant for stationarity, and we have what Conley, Hansen, Luttmer, and Scheinkman (1997) call volatility-induced stationarity.

Following the work of Aït-Sahalia (1996a and 1996b), tools for solving for stationary densities have become well-known. In particular, we know that the stationary distribution of any diffusion process $X_{t}$ is proportional to

$$
\frac{1}{\sigma(X)^{2}} \exp \left(\int_{x_{0}}^{X} \frac{2 \mu(x)}{\sigma(x)^{2}} d x\right)
$$

For the CEV model, this works out to be proportional to

$$
\frac{1}{\sigma^{2} V^{2 \gamma_{1}}} \exp \left(\frac{2 \alpha}{\sigma^{2}\left(1-2 \gamma_{1}\right)} V^{1-2 \gamma_{1}}+\frac{2 \beta}{\sigma^{2}\left(2-2 \gamma_{1}\right)} V^{2-2 \gamma_{1}}\right)
$$

where $\sigma=\sqrt{\sigma_{1}^{2}+\sigma_{2}^{2}}$. For the SQRT model, the unconditional density is proportional to the simpler

$$
\frac{1}{\sigma^{2} V} \exp \left(\frac{2 \alpha}{\sigma^{2}} \ln V+\frac{2 \beta}{\sigma^{2}} V\right)
$$

The unconditional distribution for the 2GAM model is more difficult to derive and will be computed by numerical integration. The form of the stationary density is identical in the case of reflecting boundaries, though the constant of proportionality will be slightly altered to account for the zero mass below $V^{L}$ and above $V^{U}$ (see Lewis, 2000, for a discussion).

Figures 6A to 6C plot the unconditional distributions of $V$ under the objective measure using the posterior means reported in Table 1. Constants of integration are calculated numerically, and the densities are normalized to integrate to one.

Figure 6A plots the stationary density of $V$ for the SQRT model. The solid line represents the distribution calculated over the post-crash subperiod, while the dashed line uses estimates from the whole sample. It is somewhat discomforting that both densities have significant mass vary close to zero, and that distributions fatter right tail for the whole sample coincides with an even greater amount of mass near zero. 
By comparison, Figure 6B shows that the densities for the CEV processes have virtually no mass near zero. For both sample periods, the modes of the distributions correspond to one-day volatilities of about three quarters of a percent. The steady state densities for the 2GAM model, shown in Figure 6C, are nearly identical to those of the CEV process for parameter values from the same sample period.

Figures 7A to 7C shows the ratios of each pair of densities over the two sample periods. For both samples, Figure 7A shows that the CEV density declines more quickly than the SQRT model for somewhat higher variances in the range of .0001 to .0004, corresponding to volatilities of one or two percent per day. Eventually, the CEV's right tail dominates SQRT's as $V$ becomes even larger. In the post-July 1988 subsample, a daily volatility of about three percent $\left(V_{0}=.001\right)$ is over thirty times more likely under the CEV model than it is for the SQRT model. Figure 7B demonstrates very similar behavior for the 2GAM model.

Finally, Figure 7C highlights the few differences between the 2GAM and CEV densities. As $V$ goes to zero, the 2GAM density dominates that of the CEV model, although since both are virtually zero this is of little importance. More important is the fact that the 2GAM density approaches zero slightly more quickly as $V$ gets large, which foreshadows the finding that the 2GAM model produces less kurtosis than does the CEV model.

The possibility that $V$ could become arbitrarily close to zero, were the lower bound removed, has implications for the absence of arbitrage. ${ }^{11}$ Although Heston was careful to disallow it, later studies, including this one, allow $\alpha$ and $\alpha^{*}$ to differ. Because volatility risk vanishes as $V \rightarrow 0$, any difference between $\alpha$ and $\alpha^{*}$ will cause the price of volatility risk to explode as $V \rightarrow 0$, a violation of no-arbitrage, as noted by Chernov and Ghysels (2000).

Based on Figure 6A, this is a legitimate concern for the SQRT model, which appears to approach zero very often. For the CEV and 2GAM models, however, this is extremely unlikely to be a problem, since Figures 6B and 6C shows that values of $V$ near zero are exceedingly rare. These models therefore potentially offer much greater freedom in the specification of volatility risk premia.

\footnotetext{
11 The lower bound is unnecessary for the regularity of the SQRT process or for the CEV process with $\gamma_{1}>1$, as I find here. It is imposed on these models only for consistency with the 2GAM model, which requires it.
} 


\subsubsection{Implied volatility paths}

To illustrate the kinds of implied volatility paths one might expect to see under each model, I will graph several simulated trajectories of implied volatility. Each path of $V_{t}$ is simulated using the post-July 1988 posterior means as the true parameters and represents a time series of 3,538 days, the length of the entire sample from January 1986 to January 2000. The initial value $V_{0}$ was drawn at random from the posterior density of $V_{t}$ across all $t$. I then approximate the hypothetical implied variance by computing $A+B V_{t}$ and adding on a mean-zero observation error with standard deviation $\xi\left(A+B V_{t-1}\right)$. Finally, this daily variance is converted to a daily standard deviation.

Figure 8 plots eight simulations performed under each model. The SQRT simulations on the left side are fairly uniform, displaying relatively regular and moderate fluctuations. In the middle and right hand columns, the simulations of the CEV and 2GAM models exhibit behavior more consistent with the actual data, plotted in Figure 1. Long periods of low and nonvolatile volatility are interrupted by occasional volatility spikes, which tend to die out quickly, the same characteristic that Schwert (1990a) noted of S\&P 500 index volatilities around the crash of October 1987.

This fast decay might be somewhat surprising since the values of $\beta$ used in the CEV and 2GAM simulations are extremely close to zero, which in linear Gaussian models would correspond to autoregressive coefficients in excess of .998. For nonlinear models, however, Conley, Hansen, Luttmer, and Scheinkman (1997, p. 532) argue that focusing on the drift alone "abstracts from the role of uncertainty," and they demonstrate that mean reversion can be due to either the diffusion or the drift. Intuitively, when the variance elasticity is large, then large movements in volatility are likely when volatility is high. When the move is upward, the volatility of volatility increases even more, furthering the chance of another large move. But when these movements are downward, the volatility of the next movement in volatility is reduced dramatically, and the process tends to stay down, sometimes for very long periods.

\subsubsection{The price/variance correlation}

For the 2GAM model, Table 1 showed that $\gamma_{1}>\gamma_{2}$ with extremely high posterior probability. Since $\sigma_{1}<0$, the effect of this is to make the correlation between price and variance processes inversely related to the level of instantaneous variance. This is clear from (5). 
Figure 9 shows this correlation as a function of instantaneous volatility. On a typical day, the volatility of the OEX is just under one percent per day, and the figure shows that all models imply relatively similar correlations. It is when markets become turbulent that the correlation implied by the 2GAM model becomes even more negative. In the most extreme cases, when daily volatility exceeds $3 \%$, this correlation is fairly close to negative one. Through this mechanism, extreme shocks to volatility are likely to be associated with large negative returns. This will substantially increase the skewness of returns under the 2GAM model relative to the CEV model, as will be shown below.

\subsection{Skewness and kurtosis of returns}

The ability to generate realistic higher moments in stock returns is one of a stochastic volatility model's most crucial requirements. For several reasons, I focus not on the population moments implied by the various models, but on the distributions of sample moments generated by each model over several relevant sample sizes and sampling frequencies. These distributions supply a more informative description of the return characteristics implied by each model than do population moments since they address more directly what values of higher moments are likely to be found in a finite sample of data. ${ }^{12}$ For this reason, the focus on sample moments offers a more parallel basis to compare observed and model-implied moments.

In this section I examine whether the distribution of sample moments and crash frequencies implied by the models previously estimated are consistent with the observed data. To account for the uncertainty of the parameter estimates, distributions of sample moments are computed using many different draws from the posterior distribution of the parameter vector. Specifically, I draw 5,000 parameter vectors at random from each model's posterior distribution. For each parameter draw, I simulate both a 13-year and a 115-year sample of returns data, starting with a randomly-drawn initial level of $V .{ }^{13}$ For each sample I compute skewness and kurtosis for daily, weekly, and monthly returns. Various percentiles of these distributions are reported in Tables 3 and 4.

\footnotetext{
12 For example, they will reveal whether higher moments are driven primarily by large returns occurring somewhat infrequently (a high median, low variance distribution of sample kurtosis) or by enormous returns occurring with minute probability (a low median, high variance, and right skewed distribution of sample kurtosis).

${ }^{13}$ I draw values of $V_{0}$ at random from the unconditional densities plotted previously by discretizing the space of instantaneous variances into 10,000 evenly-spaced points ranging from $10^{-8}$ to 1 . Values of $V_{0}$ are then chosen by drawing a uniform random number on the interval $(0,1)$ and evaluating the inverse cdf of the discretized distribution.
} 
For comparison, I also include the skewness and kurtosis generated by Pan's (2000) jumpdiffusion model (her "SVJ" model), a model that combines the square root variance process with a state-dependent Poisson jump. Because no posterior distribution is available for this model, distributions of sample moments are computed using the point estimates reported in Table 1 of her paper. ${ }^{14}$

The simulated distribution for the SQRT, CEV, and 2GAM models represent a type of predictive distribution that is not conditioned on the terminal values of the sample data, since the initial value of volatility in each simulation is drawn from the unconditional distribution of the process rather than the filtered distribution of volatility on the last day of the sample. The simulations for Pan's model, since they are based on a single set of point estimates, generate what is commonly called a parametric bootstrap distribution.

Actual sample moments are computed over three sample periods. The shortest sample period corresponds to the post-crash period used in estimating the model. A slightly longer sample starts in January 1986, and therefore includes the crash of '87. A much longer and somewhat different sample covers January 1885 through June 2000. Returns data prior to 1962 come from Schwert (1990b), and the later portion is the S\&P 500 return from CRSP. ${ }^{15}$ Multiperiod sample moments are calculated using overlapping returns.

Table 3 compares model-implied distributions of 13-year sample moments with actual sample moments calculated over the $1986-2000$ and $1988-2000$ periods. ${ }^{16}$ It is immediately apparent that the 2GAM and CEV models typically generate far greater deviations from normality than do the SQRT model or even the model that incorporates jumps. The table shows that the 1986-2000 sample skewness and kurtosis lie far beyond the $1^{s t}$ and $99^{\text {th }}$ percentiles of the distributions implied by the SQRT model. Even for the relatively calm post-crash sample, the kurtosis of daily returns is still too high to have likely resulted from the SQRT model.

For daily returns, the CEV model produces a much higher median kurtosis of 7.14, only slightly below the kurtosis of 8.06 observed in the post-crash sample. Even the extreme kurtosis of 49.23 observed in the 1986-2000 sample is not entirely inconsistent with the CEV model, as this value lies in between the $95^{t h}$ and $99^{\text {th }}$ percentiles of the model-implied distribution. In matching the skewness of -2.23 observed over the 1986-2000 sample, however, the CEV

\footnotetext{
${ }^{14}$ Pan's parameter estimates come from a sample of weekly data from January 1989 to December 1996, which corresponds roughly with my post-crash sample.

${ }^{15}$ Schwert's (1990b) returns series is constructed from the Dow Jones index until 1928, and the S\&P composite index thereafter.

16 The average length of these two samples is about 13 years.
} 
model falls short, generating skewness values below -1.48 only one percent of the time. The 2GAM model, in contrast, is able to produce substantially more negative skewness with a more moderate level of kurtosis, a result of its time-varying correlation between returns and volatility.

For weekly and monthly returns, both the CEV and 2GAM models continue to perform well, with actual sample moments between the model-implied $5^{\text {th }}$ and $95^{\text {th }}$ percentiles in every case but one.

While Pan's SVJ model typically produces significantly fatter-tailed samples than does the SQRT model (on which it is based), the model-implied distributions of skewness and kurtosis are incompatible with the values observed over the 1986-2000 sample period.

The same simulations can be examined to check the impact of imposing upper and lower bounds on the variance process. For the SQRT model, the upper bound $V^{U}=1$ was never reached, but the lower bound $V^{L}=10^{-8}$ was hit at least once quite often, on 1510 out of the 5000 simulated paths, demonstrating a certain unnatural implication of the SQRT model that many returns are extremely small in absolute value. The lower bound was not touched by any of the CEV paths, while it was hit by 17 of the 5000 2GAM variance paths. The upper bound, meanwhile, was hit six times by the CEV model and two times by the 2GAM model, consistent with the extreme tail behavior of these two models.

Table 4 repeats the analysis with a 115-year data sample. The results are fairly similar, with both the SQRT model and Pan's SVJ model incapable of generating the degree of kurtosis required to fit the data, but faring better in matching skewness. The CEV model performs well in general, bracketing all sample moments within the $5^{\text {th }}$ and $95^{\text {th }}$ percentiles of its posteriors. Slightly worse is the 2GAM model, since it is incapable of generating the positive monthly skewness surprisingly observed over the extended sample.

Table 4 shows that model-implied sample skewness and kurtosis can occasionally be even more extreme over longer sample periods. The CEV model, for example, produces a kurtosis of 685 or more in one percent of the 115-year-long samples. A sample kurtosis this extreme would have been generated, for example, had the crash of October 1987 been a one-day drop of 75 percent rather than 21 percent and the remainder of the 115 years of data been identical. A kurtosis of 210, generated by the 2GAM model in one out of 100 samples, would have resulted from a 53 percent drop. These extreme sample moments emphasize the ability of the CEV and 2GAM models to produce very large returns with nonzero probability.

The frequency with which each model generates large negative returns, or market crashes, 
is further investigated in Table 5. Using the same simulations as in Table 4, I calculate the number of returns in each simulated 115-year sample that were below -5, -10, and -20 percent. Table 5 contains various percentiles of the distributions of these crash frequencies, along with the actual number of stock market crashes observed in sample. As before, the distributions calculated for the CEV and 2GAM models are surprisingly consistent with the actual data, with actual crash frequencies always between the $5^{\text {th }}$ and $95^{\text {th }}$ percentiles of the model-implied distributions. The SQRT model again performs poorly, never generating a daily return below -20 percent and very rarely never below -10 percent, although it still generates more frequent crashes than Pan's SVJ model. Although the jump component increases the non-normality of returns, Pan's estimates imply a lower return variance than does the SQRT model, making very large returns rare.

While each model's implications for conditional moments is as interesting as their implications for unconditional moments, the calculation of conditional sample moments is not straightforward. I therefore examine conditional moments indirectly through their impact on options prices.

\subsection{Option pricing}

If the CEV and 2GAM models produce substantially greater non-normality in returns, they should have an advantage over the SQRT model in explaining the volatility smile, or the tendency of options far from the money to sell at premiums relative to a Black-Scholes benchmark calculated using the implied volatility of an at-the-money option. I will therefore examine how the different models price puts and calls of different maturities with varying degrees of moneyness. I calculate these prices by simulation under the risk-neutral measure, using the post-July 1988 posterior means as the parameter values for simulation. For simplicity, I assume that the interest and dividend rates are zero, so that the risk-neutralized stock price process is a martingale.

Each option price is calculated from 100,000 simulations assuming an initial stock price of $\$ 100$. Two variance reduction techniques are used: antithetic variables and conditional simulations, whereby only $V_{t}$ is simulated. The latter technique is developed by Willard (1997) and implemented recently by Poteshman (1998). ${ }^{17}$ For the 2GAM model, Willard's method is

\footnotetext{
17 Willard (1997) shows that conditional on a simulated path of $V$, the terminal stock price has a lognormal distribution, even if the price and variance paths are correlated. The price of a call option may therefore be computed by averaging Black-Scholes call prices generated by each of these lognormals.
} 
inapplicable since the price/variance correlation is time-varying, so I instead use the extension of Romano and Touzi (1997).

Table 6 shows the prices of puts and calls generated by each model for a variety of strike prices, times to maturity, and initial variance levels. To highlight the difference between the models, no in-the-money options were included. Standard errors of the put and call prices are not reported but are less than two percent of the option price for all options whose values exceed .0001 .

The CEV and 2GAM models tends to produce high option prices for at least two reasons. First, the drift of the variance process under the risk-neutral measure is more positive for the CEV model than it is for the SQRT model, making all options priced under the CEV model more valuable. Second, the CEV model generates fatter tails, making options that are far from at-the-money especially expensive.

For the shortest term options, the first effect is minor and all models generate comparable prices for options that are approximately at-the-money. The main differences between the two are for options that are far from at-the-money. Deep out-of-the-money put options are valued higher by the CEV and 2GAM models than by the SQRT model. In some cases these options are ten times more expensive under the CEV model and almost 50 times more expensive under the 2GAM model. In contrast, the deep out-of-the-money call options are sometimes more expensive under the SQRT model, reflecting the positive skewness of returns for the SQRT model at short horizons.

For longer term options, the large positive drift in the CEV variance process implies very expensive options. As with shorter term options, however, the biggest differences between the CEV and SQRT models are found in deep OTM puts, where the CEV model's fat tails lead to even higher prices.

All of these patterns are consistent with the greater kurtosis and more negative skewness generated by the CEV model in Tables 3 and 4 . Furthermore, they would appear to help explain the patterns of implied volatility observed in equity index options markets, where deep out-ofthe-money puts are extremely expensive relative to the put prices computed using the at-themoney implied volatilities.

To determine whether the implied volatility smiles generated by the CEV and SQRT models are consistent with options price data, I engage in a preliminary analysis using closing prices of S\&P 100 equity options from July 1988 through August 1996. Black-Scholes implied volatil- 
ities are calculated using the method of Aït-Sahalia and Lo (1998). Since this method is only applicable to European-style options, the results here are open to criticism. It will be apparent below, however, that the early exercise premium would have to be extremely large to alter the results. In addition, the qualitative results are identical if S\&P 500 options are used instead, and these options are European.

Using this method, simultaneously-observed puts and calls with the same strike price and maturity are used to infer the implied index forward price. This circumvents the need for an accurate dividend forecast and a concurrent value for the true value of the index, but allows only one implied volatility to be extracted from every pair of options. An interest rate is still needed to compute the implied volatility, so I use the Treasury bill rate (from the Federal Reserve's H15 release) with maturity closest to the option's expiration date.

I define "at-the-money" as the equality of the strike price and the implied forward price. I discard all option prices that are not part of matched pairs as well as all option prices recorded on days without at least two matched pairs. On each day, at-the-money implied volatilities for each maturity are calculated by interpolating (or occasionally extrapolating) the corresponding volatility smiles on that day.

I compute the difference between each matched pair's implied volatility and the contemporaneous at-the-money implied volatility for the same maturity. This difference, the "smile magnitude," will be plotted against a normalized strike price, which is equal to the true strike price over the implied forward price minus one. Looking at the difference between two implied volatilities calculated from the same model should reduce the extent with which the differences between the models are driven simply by different levels of risk-neutral drift.

Although we should expect this measure of the volatility smile to depend on both the time to maturity of the option and the level of at-the-money implied volatility, I group data only by the former. Three maturity groups are considered: (a) options with between 21 and 23 days to expiration, (b) options with between 64 and 68 days to expiration, and (c) options with between 120 and 144 days to expiration. ${ }^{18}$

The observed smile magnitudes (dots) are plotted in Figure 10 along with the volatility smiles generated by the CEV (solid line), the 2GAM (dashed line), and SQRT (dot-dash line) models. Subplots on the left side assume an initial level of instantaneous variance equal to

\footnotetext{
${ }^{18}$ In an earlier version of this paper, I examined the implied volatility smile of 5-day options as well. After observing that the bulk of the out-of-the-money option prices were no larger than a single tick, I excluded all very short-term options from the paper.
} 
.0001 , while those on the right assume $V_{0}=.001$. In general, the model-implied smiles for 22day (one-month) options are far less severe than those observed in the data. For three-month options, the fit of the CEV and 2GAM models is somewhat better, while for six-month options the fit is quite good. In all cases, the SQRT model is clearly inadequate in explaining most options prices.

\section{Conclusion}

As opposed to the GARCH literature on stock volatility, in which new models are primarily introduced to explain previously unnoticed features of price dynamics, the continuous-time literature has tended to favor models that generate closed form solutions for derivative prices. While such an emphasis is natural given the typical uses of these models, the inability of popular models to generate realistic price dynamics limits their usefulness.

The new models introduced in this paper have obvious advantages over the square root model of Heston (1993) and others. While the square root model does produce non-Gaussian

returns, the skewness and kurtosis it generates are far too small to be consistent with equity index returns. In fact, when volatility does grow large under the square root model, the nonGaussian features of the return distribution disappear almost completely.

The higher order moments that were reported for the CEV and 2GAM models were implied by parameters estimated over a sample from July 1988 to January 2000. While this is by no means a period of low market volatility, it is a period that excludes the crash of 1987 and the turbulent markets that followed the crash of 1929. Nevertheless, the skewness and kurtosis generated by the more general models matched the sample moments over longer periods remarkably well. Therefore, jumps appear unnecessary as a device to increase unconditional skewness and kurtosis of returns.

Unfortunately, while the option prices and volatility smiles generated by the CEV and 2GAM models are in many cases much different from the prices implied by the square root model, they are still inconsistent with the volatility smile consistently observed in short-term equity index options.

Although these option prices remain somewhat of a puzzle, many other aspects of the data are less perplexing. The results of this paper suggest a simple link between the observed intertemporal patterns in volatility and the existence of very rare and very large market crashes. 
When volatility is low, it tends to stay low, sometimes over extremely long periods, since its drift is near zero and its own variance is small. When eventually there is a large positive shock to the volatility process, volatility itself rapidly becomes more volatile, and the probability of a larger subsequent rise in volatility increases dramatically. Successive positive shocks to volatility can therefore compound rapidly, and the eventual occurrence of such shocks makes the extreme return a rare but regular event. When the degree of negative correlation between prices and volatility increases, these extreme returns, more likely than not, are stock market crashes. Following the crash, these high volatility states tend to die out quickly because the mean reversion in volatility is itself volatility-induced.

While there remain unanswered questions regarding long memory in volatility, parameter constancy, and options pricing, the models developed here show clear potential as simple yet much more realistic generalizations of existing models. 


\section{Appendices}

\section{A Derivation of expected average variance}

Suppose the dynamics of $V_{t}$ are described by the SDE

$$
d V_{t}=\left(\alpha+\beta V_{t}\right) d t+\sigma\left(V_{t}\right) d B
$$

and that the stochastic integral

$$
\int_{\mathcal{S}=t}^{T} \sigma\left(V_{\mathcal{S}}\right) d B_{\mathcal{S}}
$$

is a martingale ${ }^{19}$, where $t<T$. Then we have, using the martingale property and Fubini's theorem,

$$
\begin{aligned}
E_{t}\left[V_{T}\right] & =V_{t}+E_{t}\left[\int_{s=t}^{T}\left(\alpha+\beta V_{s}\right) d s\right]+E_{t}\left[\int_{s=t}^{T} \sigma\left(V_{s}\right) d B_{s}\right] \\
& =V_{t}+\int_{s=t}^{T}\left(\alpha+\beta E_{t}\left[V_{s}\right]\right) d s
\end{aligned}
$$

Therefore $f(s)=E_{t}\left[V_{s}\right]$ solves the ordinary differential equation

$$
f^{\prime}(s)=\alpha+\beta f(s)
$$

with boundary condition $f(t)=V_{t}$. This solution is

$$
f(s)=-\frac{\alpha}{\beta}\left(1-e^{\beta(s-t)}\right)+V_{t} e^{\beta(s-t)} .
$$

Expected average variance is given by

$$
\begin{aligned}
E_{t}\left[\bar{V}_{t, t+\tau}\right] & =E_{t}\left[\frac{1}{\tau} \int_{s=t}^{t+\tau} V_{s} d s\right] \\
& =\frac{1}{\tau} \int_{s=t}^{t+\tau} E_{t}\left[V_{s}\right] d s \\
& =\frac{1}{\tau} \int_{s=t}^{t+\tau}\left(-\frac{\alpha}{\beta}\left(1-e^{\beta(s-t)}\right)+V_{t} e^{\beta(s-t)}\right) d s \\
& =A+B V_{t}
\end{aligned}
$$

where

$$
B=\frac{1}{\beta \tau}\left(e^{\beta \tau}-1\right) \text { and } A=-\frac{\alpha}{\beta}(1-B) .
$$

\footnotetext{
${ }^{19}$ This is guaranteed by the upper and lower bounds on the variance process.
} 


\section{B Drawing the parameters}

In this appendix I describe draws of the four parameter blocks, $\theta^{(1)}$ through $\theta^{(4)}$, given the entire set of high-frequency stock prices and variances, written in shorthand as $S$ and $V$. As an illustration, consider the draw of $\theta^{(3)}$. We would like to draw from the distribution

$$
p\left(\theta^{(3)} \mid \theta^{(1)}, \theta^{(2)}, \theta^{(4)}, S, V\right)
$$

which is, by Bayes rule, proportional to

$$
p\left(S, V \mid \theta^{(1)}, \theta^{(2)}, \theta^{(3)}, \theta^{(4)}\right) p\left(\theta^{(3)}\right)
$$

The second term is the prior. The first term, the likelihood function, can be decomposed as the likelihood of the initial observation times the likelihood of all subsequent observations, or

$$
p\left(S, V \mid \theta^{(1)}, \theta^{(2)}, \theta^{(3)}, \theta^{(4)}\right)=p\left(S_{0}, V_{0} \mid \theta^{(1)}, \theta^{(2)}, \theta^{(3)}, \theta^{(4)}\right) p\left(S, V \mid \theta^{(1)}, \theta^{(2)}, \theta^{(3)}, \theta^{(4)}, S_{0}, V_{0}\right) .
$$

The second term is the product of Gaussian transitions, and were we to ignore the first nonGaussian term many analytical results would be available about the conditional distributions of the parameters in $\theta^{(1)}$ to $\theta^{(4)}$.

In fact, the strategy in drawing parameters will be to ignore this first term and then make a correction for it later. Specifically, we generate a candidate set of parameter draws $\theta^{(1)}$ to $\theta^{(4)}$ from distributions such as

$$
p\left(S, V \mid \theta^{(1)}, \theta^{(2)}, \theta^{(3)}, \theta^{(4)}, S_{0}, V_{0}\right) p\left(\theta^{(3)}\right)
$$

and then accept or reject the collection of draws $\theta^{(1)}$ to $\theta^{(4)}$ depending on what they imply about the missing term

$$
p\left(S_{0}, V_{0} \mid \theta^{(1)}, \theta^{(2)}, \theta^{(3)}, \theta^{(4)}\right) .
$$

Sections B. 1 to B.4 consider the draw of $\theta^{(1)}$ to $\theta^{(4)}$, while B.5 gives the criterion for acceptance.

\section{B.1 Drawing $\theta^{(1)}=\{\mu, \alpha, \beta\}$}

Given the parameters of the diffusion function, $\sigma_{1}, \sigma_{2}, \gamma_{1}$, and $\gamma_{2}$, and observation of the full price and variance paths, we can derive the conditional distribution of $\theta^{(1)}$ using the SUR methodology of Chib and Greenberg (1996). 
From the Euler approximation (9), the parameters $\mu, \alpha$, and $\beta$ can be seen as the coefficients of a bivariate linear regression with a time-varying but known residual covariance matrix. Given a flat or Gaussian prior on these parameters, the method of Chib and Greenberg (1996) can be applied directly.

\section{B.2 Drawing $\theta^{(2)}=\left\{\sigma_{1}, \sigma_{2}, \gamma_{1}, \gamma_{2}\right\}$}

The parameters $\gamma_{1}$ and $\gamma_{2}$ enter the model in a nonlinear way, so drawing from their distribution presents an additional challenge. Because little is known about the distribution of $\gamma_{1}$ and $\gamma_{2}$, I will use the Metropolis-Hastings algorithm to generate their posterior. I will assume diffuse prior information about both, or $p\left(\gamma_{1}, \gamma_{2}\right) \propto 1$. As a candidate generating density I will use a bivariate random walk, so that the candidate draw is equal to the previous draw plus two independent Gaussian innovations with mean zero and standard deviation $\zeta_{\gamma}$.

Efficiency will be improved if $\sigma_{1}$ and $\sigma_{2}$ are drawn jointly with $\gamma_{1}$ and $\gamma_{2}$, because the four parameters are likely to be highly correlated in the posterior. The candidate draws $\tilde{\sigma_{1}}$ and $\tilde{\sigma_{2}}$ will be conditioned on the candidate draw of $\tilde{\gamma_{1}}$ and $\tilde{\gamma_{2}}$. This conditional draw can be motivated by a rearrangement of the Euler approximation (9):

$$
\begin{aligned}
\frac{S_{(k+1) h}-(1+h \mu) S_{k h}}{\sqrt{h} \sqrt{V_{k h}} S_{k h}} & =\eta_{k+1}^{(1)} \\
\frac{V_{(k+1) h}-V_{k h}-h \alpha-h V_{k h} \beta}{\sqrt{h} V_{k h}^{\gamma_{2}}} & =\sigma_{1} V_{k h}^{\gamma_{1}-\gamma_{2}} \eta_{k+1}^{(1)}+\sigma_{2} \eta_{k+1}^{(2)} .
\end{aligned}
$$

Note from the first equation that given $\mu$ and the time series of $\left(S_{k}, V_{k}\right)$ the residual $\eta^{(1)}$ can be treated as an observed variable. The second equation can then be interpreted as a linear regression on an observed variable, $V_{k h}^{\gamma_{1}-\gamma_{2}} \eta_{k+1}^{(1)}$, with $\sigma_{1}$ as the regression coefficient and $\sigma_{2}$ as the standard deviation of the residual. ${ }^{20}$

With the flat prior $p\left(\sigma_{1}, \sigma_{2}\right) \propto \frac{1}{\sigma_{2}}, \sigma_{2}$ has an inverted gamma distribution and $\sigma_{1}$ has a normal distribution given $\sigma_{2}$. This bivariate distribution is used to generate candidate pairs $\left(\tilde{\sigma_{1}}, \tilde{\sigma_{2}}\right)$ given the draw of $\gamma_{1}$ and $\gamma_{2}$. The complete candidate generating density is therefore given by this IG/normal density multiplied by the bivariate normal random walk density used to draw $\gamma_{1}$ and $\gamma_{2}$. Let $f_{G}\left(\sigma_{1}, \sigma_{2}, \gamma_{1}, \gamma_{2}\right)$ denote this density.

We can calculate the true conditional distribution of $\left(\sigma_{1}, \sigma_{2}, \gamma_{1}, \gamma_{2}\right)$ up to a constant of

\footnotetext{
20 This approach is borrowed from Jacquier, Polson, and Rossi (2000).
} 
proportionality by an application of Bayes rule:

$$
p\left(\sigma_{1}, \sigma_{2}, \gamma_{1}, \gamma_{2} \mid \mu, \alpha, \beta, S, V, S_{0}, V_{0}\right) \propto p\left(S, V \mid \mu, \alpha, \beta, \sigma_{1}, \sigma_{2}, \gamma_{1}, \gamma_{2}, S_{0}, V_{0}\right) p\left(\sigma_{1}, \sigma_{2}, \gamma_{1}, \gamma_{2}\right) .
$$

The first term on the right side is the completed likelihood function - the probability of generating the full set of high frequency $S$ and $V$ given the model parameters. This likelihood is calculated using the Euler approximation and is therefore the product of bivariate Gaussian transition densities. The second term is the prior, which is proportional to $\frac{1}{\sigma_{2}}$.

The Metropolis-Hastings algorithm dictates the probability with which the candidate draws must be accepted in order for convergence to the proper distribution to occur. The probability of moving from the previous draw $\left(\sigma_{1}, \sigma_{2}, \gamma_{1}, \gamma_{2}\right)$ to the candidate draw $\left(\tilde{\sigma_{1}}, \tilde{\sigma_{2}}, \tilde{\gamma_{1}}, \tilde{\gamma_{2}}\right)$ is equal to

$$
\min \left\{\frac{p\left(S, V \mid \mu, \alpha, \beta, \tilde{\sigma_{1}}, \tilde{\sigma_{2}}, \tilde{\gamma_{1}}, \tilde{\gamma_{2}}\right) p\left(\tilde{\sigma_{1}}, \tilde{\sigma_{2}}, \tilde{\gamma_{1}}, \tilde{\gamma_{2}}\right) f_{G}\left(\sigma_{1}, \sigma_{2}, \gamma_{1}, \gamma_{2}\right)}{p\left(S, V \mid \mu, \alpha, \beta, \sigma_{1}, \sigma_{2}, \gamma_{1}, \gamma_{2}\right) p\left(\sigma_{1}, \sigma_{2}, \gamma_{1}, \gamma_{2}\right) f_{G}\left(\tilde{\sigma_{1}}, \tilde{\sigma_{2}}, \tilde{\gamma_{1}}, \tilde{\gamma_{2}}\right)}, 1\right\} .
$$

The resulting draws will converge to $p\left(\sigma_{1}, \sigma_{2}, \gamma_{1}, \gamma_{2} \mid \mu, \alpha, \beta, S, V, S_{0}, V_{0}\right)$.

\section{B.3 Drawing $\theta^{(3)}=\{A, B\}$}

Recall the assumptions about the relationship between the instantaneous and option-implied variances:

$$
I V_{t}=A+B V_{t}+\epsilon_{t}
$$

where $\epsilon_{t} \sim$ i.i.d. $N\left(0, \xi^{2} I V_{t-1}^{2}\right)$.

Conditional on the observation of the time series of $V_{t}$, this is a linear regression with heteroskedastic errors. To transform into a homoskedastic regression, define an i.i.d. $N\left(0, \xi^{2}\right)$ random variable $v_{t}$ by $v_{t}=\frac{\epsilon_{t}}{I V_{t-1}}$ and rearrange the regression equation as

$$
\frac{I V_{t}}{I V_{t-1}}=A \frac{1}{V_{t-1}} B \frac{V_{t}}{V_{t-1}}+v_{t}
$$

Given flat priors on $A$ and $B$, the posterior distribution conditional on $\xi$ is bivariate normal.

\section{B.4 Drawing $\theta^{(4)}=\{\xi\}$}

Given $A$ and $B$, a time series of homoskedastic regression residuals is defined by

$$
v_{t}=\frac{I V_{t}-A-B V_{t}}{I V_{t-1}}
$$

The parameter $\xi$ is the standard deviation of $\nu_{t}$, so given any prior of the inverted gamma form the posterior of $\xi$ is also an inverted gamma. 


\section{B.5 Incorporating the information in $V_{0}$}

Suppose we have drawn a candidate set of parameters $\theta^{(1)}$ to $\theta^{(4)}$ using the steps described in B.1 to B.4. Then these parameters are jointly drawn from a density proportional to

$$
p\left(S, V \mid \theta^{(1)}, \theta^{(2)}, \theta^{(3)}, \theta^{(4)}, S_{0}, V_{0}\right) p\left(\theta^{(1)}, \theta^{(2)}, \theta^{(3)}, \theta^{(4)}\right)
$$

which ignores the information in the initial observation $\left(S_{0}, V_{0}\right)$.

Because $S_{t}$ is obviously nonstationary, it is reasonable to assume it's stationary density is diffuse and independent of $V_{t}$. We will therefore assume

$$
p\left(S_{0}, V_{0} \mid \theta^{(1)}, \theta^{(2)}, \theta^{(3)}, \theta^{(4)}\right)=p\left(V_{0} \mid \theta^{(1)}, \theta^{(2)}, \theta^{(3)}, \theta^{(4)}\right) .
$$

The form of this density is given in section 4.4.1.

The density that we would like to draw $\theta^{(1)}$ to $\theta^{(4)}$ from takes $V_{0}$ into account, and so should be proportional to

$$
p\left(S, V \mid \theta^{(1)}, \theta^{(2)}, \theta^{(3)}, \theta^{(4)}, S_{0}, V_{0}\right) p\left(V_{0} \mid \theta^{(1)}, \theta^{(2)}, \theta^{(3)}, \theta^{(4)}\right) p\left(\theta^{(1)}, \theta^{(2)}, \theta^{(3)}, \theta^{(4)}\right) .
$$

Intuitively, we correct the previous draws of $\left(\theta^{(1)}, \theta^{(2)}, \theta^{(3)}, \theta^{(4)}\right)$ by rejecting the parameter draws that are least likely to generate $V_{0}$ in their stationary distribution. This accept/reject step is accomplished again using the Metropolis-Hastings algorithm, with the draws described in B.1 to B.4 jointly used as the candidate generator. The Metropolis-Hastings algorithm therefore dictates that the set of new draws

$$
\left(\tilde{\theta}^{(1)}, \tilde{\theta}^{(2)}, \tilde{\theta}^{(3)}, \tilde{\theta}^{(4)}\right)
$$

is accepted over the old set of draws

$$
\left(\theta^{(1)}, \theta^{(2)}, \theta^{(3)}, \theta^{(4)}\right)
$$

with probability

$$
\min \left\{\frac{p\left(V_{0} \mid \tilde{\theta}^{(1)}, \tilde{\theta}^{(2)}, \tilde{\theta}^{(3)}, \tilde{\theta}^{(4)}\right)}{p\left(V_{0} \mid \theta^{(1)}, \theta^{(2)}, \theta^{(3)}, \theta^{(4)}\right)}, 1\right\} .
$$

If the new draw is rejected, we repeat the old one.

\section{The distribution of high-frequency variance}

When $S_{t}$ and $I V_{t}$ are observed, candidate draws of $V_{t}$ are generated to be consistent with both these pieces of information in addition to the previous values $S_{t-h}$ and $V_{t-h}$. The distribution 
of these candidate draws can be decomposed as

$$
p\left(V_{t} \mid \theta^{S}, S_{t-h}, V_{t-h}, S_{t}, I V_{t}\right) \propto p\left(V_{t} \mid \theta^{S}, S_{t-h}, V_{t-h}, S_{t}\right) p\left(I V_{t} \mid \theta^{S}, V_{t}\right)
$$

The first on the right term is the conditional density of $V_{t}$ given it's previous value $V_{t-h}$ and the contemporaneous change of $S_{t}$ from $S_{t-h}$. This is simply the conditional distribution of a bivariate normal random variable, so its mean is

$$
M=V_{t-h}+h\left(\alpha+\beta V_{t-h}\right)+\rho\left(V_{t-h}\right) \frac{\sigma\left(V_{t-h}\right)}{\sqrt{V_{t-h}}}\left(S_{t}-h(1+\mu) S_{t-h}\right)
$$

and its standard deviation is

$$
\sqrt{h} \sigma\left(V_{t-h}\right) \sqrt{1-\rho^{2}\left(V_{t-h}\right)}
$$

where

$$
\rho(V)=\frac{\sigma_{1} V^{\gamma_{1}}}{\sqrt{\sigma_{1}^{2} V^{2 \gamma_{1}}+\sigma_{2}^{2} V^{2 \gamma_{2}}}}
$$

and

$$
\sigma(V)=\sqrt{\sigma_{1}^{2} V^{2 \gamma_{1}}+\sigma_{2}^{2} V^{2 \gamma_{2}}}
$$

The second term, $p\left(I V_{t} \mid \theta^{S}, V_{t}\right)$, is the Gaussian density of $I V_{t}$ given $V_{t}$. This is proportional to

$$
\exp \left(\frac{I V_{t}-A-B V_{t}}{\xi I V_{t-1}}\right)^{2}
$$

which can be rearranged as

$$
\exp \left(\frac{V_{t}-\frac{I V_{t}-A}{B}}{\frac{\xi I V_{t-1}}{B}}\right)^{2}
$$

which is also proportional to a normal density in $V_{t}$.

The density

$$
p\left(V_{t} \mid \theta^{S}, S_{t-h}, V_{t-h}, S_{t}, I V_{t}\right)
$$

is equal to the convolution of these two normals, and is therefore normal as well with mean

$$
\begin{gathered}
\left(\frac{1}{h \sigma^{2}(V)\left(1-\rho^{2}\left(V_{t-h}\right)\right)}+\frac{B^{2}}{\xi^{2} I V_{t-1}^{2}}\right)^{-1} \\
\times\left[\frac{M}{h \sigma^{2}\left(V_{t-h}\right)\left(1-\rho^{2}\left(V_{t-h}\right)\right)}+\frac{B^{2}}{\xi^{2} I V_{t-1}^{2}}\left(\frac{I V_{t}-A}{B}\right)\right]
\end{gathered}
$$

and variance

$$
\left(\frac{1}{h \sigma^{2}\left(V_{t-h}\right)\left(1-\rho^{2}\left(V_{t-h}\right)\right)}+\frac{B^{2}}{\xi^{2} I V_{t-1}^{2}}\right)^{-1} .
$$




\section{References}

[1] Aït-Sahalia, Y., 1996a, “Testing Continuous-Time Models of the Spot Interest Rate,” Review of Financial Studies 9, 385-426.

[2] Aït-Sahalia, Y., 1996b, “Nonparametric Pricing of Interest Rate Derivative Securities,” Econometrica $64,527-560$.

[3] Aït-Sahalia, Y., 1998, "Nonparametric Estimation of State-Price Densities Implicit in Financial Asset Prices," Journal of Finance 53, 499-547.

[4] Andersen, T. G., L. Benzoni, and J. Lund, 2000, "Estimating Jump-Diffusions for Equity Returns," working paper.

[5] Bakshi, G., C. Cao, and Z. Chen, 1997, "Empirical Performance of Alternative Option Pricing Models," Journal of Finance 52, 2003-2049.

[6] Bakshi, G., C. Cao, and Z. Chen, 2000, "Pricing and Hedging Long-Term Options,” Journal of Econometrics 94, 277-318.

[7] Ball, C. A., and W. N. Torous, 1985, “On Jumps in Common Stock Prices and their Impact on Call Option Pricing," Journal of Finance 40, 155-173.

[8] Bates, D. S., 2000, "Post-'87 Crash Fears in the S\&P 500 Futures Option Market," Journal of Econometrics 94, 181-238.

[9] Benzoni, L., 1999, "Pricing Options Under Stochastic Volatility: An Econometric Analysis,” working paper.

[10] Bergman, Y. Z., G. D. Grundy, and Z. Wiener, 1996, “General Properties of Option Prices,” Journal of Finance 51, 1573-1610.

[11] Black, F., and M. Scholes, 1973, “The Pricing of Options and Corporate Liabilities,” Journal of Political Economy 81, 637-659.

[12] Blair, B., S. Poon, and S. J. Taylor, 1999, "Forecasting S\&P 100 Volatility: The Incremental Information Content of Implied Volatilities and High Frequency Index Returns,” working paper. 
[13] Bollerslev, T., 1986, “Generalized Autoregressive Conditional Heteroskedasticity,” Journal of Econometrics 31, 307-327.

[14] Brandt, M., and P. Santa-Clara, 1999, "Simulated Likelihood Estimation of Multivariate Diffusions with an Application to Interest Rates and Exchange Rates with Stochastic Volatility,” working paper.

[15] Breeden, D., and R. Litzenberger, 1978, "Prices of State-Contingent Claims Implicit in Options Prices," Journal of Business 51, 621-651.

[16] Casella, G., and E. I. George, 1992, “Explaining the Gibbs Sampler,” American Statistician $46,167-174$.

[17] Chacko, G., and L. M. Viceira, 1999, "Spectral GMM Estimation of Continuous-Time Processes," working paper.

[18] Chan, K. C., G. A. Karolyi, F. A. Longstaff, and A. B. Sanders, 1992, “An Empirical Comparison of Alternative Models of the Short-Term Interest Rate,” Journal of Finance 47, 12091227.

[19] Chernov, M., and E. Ghysels, 1999, "Estimation of Stochastic Volatility Models for the Purpose of Options Pricing," working paper.

[20] Chernov, M., and E. Ghysels, 2000, “A Study towards a Unified Approach to the Joint Estimation of Objective and Risk Neutral Measures for the Purpose of Options Valuation," Journal of Financial Economics, 56, 407-458.

[21] Chib, S., and E. Greenberg, 1995, "Understanding the Metropolis-Hastings Algorithm," American Statistician 49, 327-335.

[22] Chib, S., and E. Greenberg, 1996, "Markov Chain Monte Carlo Simulation Methods in Econometrics," Econometric Theory 12, 409-431.

[23] Christensen, B. J., and N. R. Prabhala, 1998, “The Relation between Implied and Realized Volatility," Journal of Financial Economics 50, 125-150.

[24] Conley, T. G., L. P. Hansen, E. G. J. Luttmer, and J. A. Scheinkman, 1997, "Short-Term Interest Rates as Subordinated Diffusions," Review of Financial Studies 10, 525-577. 
[25] Duffie, D., J. Pan, and K. Singleton, 1999, “Transform Analysis and Asset Pricing for Affine Jump-Diffusions," Econometrica, forthcoming.

[26] Engle, R. F., 1982, “Autoregressive Conditional Heteroskedasticity with Estimates of the Variance of UK Inflation,” Econometrica 50, 987-1008.

[27] Fleming, J., B. Ostdiek, and R. E. Whaley, 1995, "Predicting Stock Market Volatility: A New Measure,” Journal of Futures Markets 15, 265-302.

[28] Gallant, R., and G. Tauchen, 1996, “Which Moments to Match?,” Journal of Econometric Theory 12, 657-681.

[29] Gallant, R., and G. Tauchen, 1997, "Estimation of Continuous-Time Models for Stock Returns and Interest Rates," Macroeconomic Dynamics 1, 137-168.

[30] Harvey, C. R., and R. E. Whaley, 1991, “Dividends and S\&P 100 Index Option Valuation,” Journal of Futures Markets 12, 123-137.

[31] Hentschel, L., 1995, "All in the Family: Nesting Symmetric and Asymmetric GARCH Models,” Journal of Financial Economics 39, 71-104.

[32] Heston, S., 1993, “A Closed-Form Solution for Options with Stochastic Volatility with Applications to Bond and Currency Options," Review of Financial Studies 6, 327-343.

[33] Hull, J., and A. White, 1987, “The Pricing of Options on Assets with Stochastic Volatilities,” Journal of Finance 42, 281-300.

[34] Jacquier, E., N. G. Polson, and P. E. Rossi, 1994, "Bayesian Analysis of Stochastic Volatility Models," Journal of Business and Economic Statistics 12, 371-389.

[35] Jacquier, E., N. G. Polson, and P. E. Rossi, 1999, "Stochastic Volatility: Univariate and Multivariate Extensions," working paper.

[36] Jacquier, E., N. G. Polson, and P. E. Rossi, 2000, "Bayesian Analysis of Stochastic Volatility with Leverage Effect and Fat Tails,” working paper.

[37] Jarrow, R., and A. Rudd, 1982, “Approximate Option Valuation for Arbitrary Stochastic Processes," Journal of Financial Economics 10, 347-369. 
[38] Jiang, G., and P. van der Sluis, 1998, "Pricing Stock Options Under Stochastic Volatility and Interest Rates with Efficient Method of Moments Estimation,” working paper.

[39] Jones, C. S., 1998, "Bayesian Estimation of Continuous-Time Finance Models,” working paper.

[40] Jones, C. S., 2000, “Nonlinear Mean Reversion in the Short-Term Interest Rate,” working paper.

[41] Jorion, P., 1989, “On Jump Processes in the Foreign Exchange and Stock Markets,” Review of Financial Studies 1, 427-445.

[42] Kloeden, P. E., and E. Platen, 1992, Numerical Solution of Stochastic Differential Equations, Springer-Verlag, New York.

[43] Lewis, A. L., 2000, Option Valuation Under Stochastic Volatility, Finance Press, Newport Beach, CA.

[44] Nelson, D. B., 1991, "Conditional Heteroskedasticity in Asset Returns: A New Approach,” Econometrica 59, 347-370.

[45] Pagan, A. R., and G. W. Schwert, 1990, “Alternative Models for Conditional Stock Volatility,” Journal of Econometrics 45, 267-290.

[46] Pan, J., 2000, “'Integrated’ Time-Series Analysis of Spot and Options Prices,” working paper.

[47] Pedersen, A. R., 1995, “A New Approach to Maximum Likelihood Estimation for Stochastic Differential Equations Based on Discrete Observations," Scandinavian Journal of Statistics $22,55-71$.

[48] Poteshman, A. M., 1998, "Estimating a General Stochastic Variance Model from Option Prices," working paper.

[49] Romano, Marc, and Nizar Touzi, 1997, "Contingent Claims and Market Completeness in a Stochastic Volatility Model," Mathematical Finance 7, 399-412.

[50] Schwert, G. W., 1990a, “Stock Volatility and the Crash of '87," Review of Financial Studies 3, 77-102. 
[51] Schwert, G. W., 1990b, “Indexes of U. S. Stock Prices from 1802 to 1987," Journal of Business 63, 399-426.

[52] Scott, L. O., 1997, "Pricing Stock Options in a Jump-Diffusion Model with Stochastic Volatility and Interest Rates: Applications of Fourier Inversion Methods," Mathematical Finance 7, 413-426.

[53] Tanner, M. A., and W. H. Wong, 1987, “The Calculation of Posterior Distributions by Data Augmentation," Journal of the American Statistical Association 82, 528-549.

[54] Whaley, R. E., 1993, “Derivatives on Market Volatility: Hedging Tools Long Overdue,” Journal of Derivatives 1, 71-84.

[55] Willard, G., 1997, "Calculating Prices and Sensitivities for Path-Independent Derivative Securities in Multifactor Models," Journal of Derivatives 5, 45-61.

[56] Zellner, A., 1971, An Introduction to Bayesian Inference in Econometrics, John Wiley, New York. 
Table 1

\section{Posterior Means and Standard Deviations}

Posterior means and standard deviations (in parentheses) for each model are computed numerically from Markov chain Monte Carlo output as described in Section 4. Each model is estimated over two sample periods, one that includes the crash of 1987 and one that does not. The 19862000 sample extends from January 2, 1986 to June 2, 2000, while the "post-crash" 1988-2000 sample does not begin until July 1, 1988.

\begin{tabular}{|c|c|c|c|c|c|c|}
\hline Parameter & $\begin{array}{c}\text { SQRT } \\
1986-2000\end{array}$ & $\begin{array}{c}\text { SQRT } \\
1988-2000\end{array}$ & $\begin{array}{c}\text { CEV } \\
1986-2000\end{array}$ & $\begin{array}{c}\text { CEV } \\
1988-2000\end{array}$ & $\begin{array}{c}\text { 2GAM } \\
1986-2000\end{array}$ & $\begin{array}{c}\text { 2GAM } \\
1988-2000\end{array}$ \\
\hline$\mu$ & $\begin{array}{c}2.37 \times 10^{-4} \\
\left(1.23 \times 10^{-4}\right)\end{array}$ & $\begin{array}{c}3.24 \times 10^{-4} \\
\left(1.34 \times 10^{-4}\right)\end{array}$ & $\begin{array}{c}1.39 \times 10^{-4} \\
\left(1.90 \times 10^{-4}\right)\end{array}$ & $\begin{array}{c}4.71 \times 10^{-4} \\
\left(1.40 \times 10^{-4}\right)\end{array}$ & $\begin{array}{c}3.62 \times 10^{-4} \\
\left(1.84 \times 10^{-4}\right)\end{array}$ & $\begin{array}{r}4.84 \times 10^{-4} \\
(1.38) \times 10^{-4}\end{array}$ \\
\hline$\alpha$ & $\begin{array}{c}6.01 \times 10^{-6} \\
\left(0.44 \times 10^{-6}\right)\end{array}$ & $\begin{array}{c}2.02 \times 10^{-6} \\
\left(0.27 \times 10^{-6}\right)\end{array}$ & $\begin{array}{c}5.87 \times 10^{-7} \\
\left(1.84 \times 10^{-7}\right)\end{array}$ & $\begin{array}{c}5.38 \times 10^{-7} \\
\left(1.42 \times 10^{-7}\right)\end{array}$ & $\begin{array}{c}2.03 \times 10^{-7} \\
\left(1.54 \times 10^{-7}\right)\end{array}$ & $\begin{array}{c}5.64 \times 10^{-7} \\
\left(1.44 \times 10^{-7}\right)\end{array}$ \\
\hline$\beta$ & $\begin{array}{l}-3.73 \times 10^{-2} \\
\left(0.47 \times 10^{-2}\right)\end{array}$ & $\begin{array}{l}-1.56 \times 10^{-2} \\
\left(0.29 \times 10^{-2}\right)\end{array}$ & $\begin{array}{c}2.02 \times 10^{-2} \\
\left(0.30 \times 10^{-2}\right)\end{array}$ & $\begin{array}{l}-0.14 \times 10^{-2} \\
\left(0.32 \times 10^{-2}\right)\end{array}$ & $\begin{array}{c}2.24 \times 10^{-2} \\
\left(0.29 \times 10^{-2}\right)\end{array}$ & $\begin{array}{l}-0.09 \times 10^{-2} \\
\left(0.31 \times 10^{-2}\right)\end{array}$ \\
\hline$\sigma_{1}$ & $\begin{array}{l}-1.34 \times 10^{-3} \\
\left(0.07 \times 10^{-3}\right)\end{array}$ & $\begin{array}{l}-1.07 \times 10^{-3} \\
\left(0.03 \times 10^{-3}\right)\end{array}$ & $\begin{array}{l}-4.94 \times 10^{-1} \\
\left(0.50 \times 10^{-1}\right)\end{array}$ & $\begin{array}{l}-6.61 \times 10^{-1} \\
\left(1.57 \times 10^{-1}\right)\end{array}$ & $\begin{array}{l}-8.77 \\
(0.92)\end{array}$ & $\begin{array}{l}-4.66 \\
(1.59)\end{array}$ \\
\hline$\sigma_{2}$ & $\begin{array}{c}2.75 \times 10^{-3} \\
\left(0.05 \times 10^{-3}\right)\end{array}$ & $\begin{array}{c}1.15 \times 10^{-3} \\
\left(0.03 \times 10^{-3}\right)\end{array}$ & $\begin{array}{c}2.88 \times 10^{-1} \\
\left(0.28 \times 10^{-1}\right)\end{array}$ & $\begin{array}{c}6.26 \times 10^{-1} \\
\left(1.48 \times 10^{-1}\right)\end{array}$ & $\begin{array}{c}3.64 \times 10^{-2} \\
\left(0.61 \times 10^{-2}\right)\end{array}$ & $\begin{array}{c}3.68 \times 10^{-2} \\
\left(1.48 \times 10^{-2}\right)\end{array}$ \\
\hline$\gamma_{1}$ & $\begin{array}{l}0.50 \\
\mathrm{~N} / \mathrm{A}\end{array}$ & $\begin{array}{l}0.50 \\
\mathrm{~N} / \mathrm{A}\end{array}$ & $\begin{array}{c}1.12 \\
(0.01)\end{array}$ & $\begin{array}{c}1.22 \\
(0.03)\end{array}$ & $\begin{array}{l}1.48 \\
(0.02)\end{array}$ & $\begin{array}{l}1.41 \\
(0.04)\end{array}$ \\
\hline$\gamma_{2}$ & & & & & $\begin{array}{c}0.87 \\
(0.02)\end{array}$ & $\begin{array}{c}0.91 \\
(0.04)\end{array}$ \\
\hline$A$ & $\begin{array}{c}4.07 \times 10^{-5} \\
\left(0.07 \times 10^{-5}\right)\end{array}$ & $\begin{array}{c}3.07 \times 10^{-5} \\
\left(0.05 \times 10^{-5}\right)\end{array}$ & $\begin{array}{c}3.59 \times 10^{-5} \\
\left(0.07 \times 10^{-5}\right)\end{array}$ & $\begin{array}{c}0.11 \times 10^{-5} \\
\left(0.09 \times 10^{-5}\right)\end{array}$ & $\begin{array}{c}3.70 \times 10^{-5} \\
\left(0.07 \times 10^{-5}\right)\end{array}$ & $\begin{array}{c}1.00 \times 10^{-5} \\
\left(0.34 \times 10^{-5}\right)\end{array}$ \\
\hline$B$ & $\begin{array}{c}0.92 \\
(0.01)\end{array}$ & $\begin{array}{c}1.12 \\
(0.02)\end{array}$ & $\begin{array}{c}0.41 \\
(0.01)\end{array}$ & $\begin{array}{c}1.77 \\
(0.04)\end{array}$ & $\begin{array}{c}0.45 \\
(0.01)\end{array}$ & $\begin{array}{c}1.64 \\
(0.08)\end{array}$ \\
\hline$\xi$ & $\begin{array}{c}1.75 \times 10^{-1} \\
\left(0.03 \times 10^{-1}\right)\end{array}$ & $\begin{array}{c}0.43 \times 10^{-1} \\
\left(0.02 \times 10^{-1}\right)\end{array}$ & $\begin{array}{c}0.66 \times 10^{-1} \\
\left(0.02 \times 10^{-1}\right)\end{array}$ & $\begin{array}{c}0.35 \times 10^{-1} \\
\left(0.01 \times 10^{-1}\right)\end{array}$ & $\begin{array}{c}0.65 \times 10^{-1} \\
\left(0.02 \times 10^{-1}\right)\end{array}$ & $\begin{array}{c}0.35 \times 10^{-1} \\
\left(0.01 \times 10^{-1}\right)\end{array}$ \\
\hline$\rho(.0001)$ & $\begin{array}{l}-0.44 \\
(0.02)\end{array}$ & $\begin{array}{l}-0.68 \\
(0.01)\end{array}$ & $\begin{array}{l}-0.86 \\
(0.01)\end{array}$ & $\begin{array}{l}-0.73 \\
(0.01)\end{array}$ & $\begin{array}{l}-0.67 \\
(0.02)\end{array}$ & $\begin{array}{l}-0.78 \\
(0.01)\end{array}$ \\
\hline$\sigma(.0001)$ & $\begin{array}{c}3.06 \times 10^{-5} \\
\left(0.06 \times 10^{-5}\right)\end{array}$ & $\begin{array}{c}1.57 \times 10^{-5} \\
\left(0.03 \times 10^{-5}\right)\end{array}$ & $\begin{array}{c}1.88 \times 10^{-5} \\
\left(0.05 \times 10^{-5}\right)\end{array}$ & $\begin{array}{c}1.19 \times 10^{-5} \\
\left(0.02 \times 10^{-5}\right)\end{array}$ & $\begin{array}{c}1.57 \times 10^{-5} \\
\left(0.04 \times 10^{-5}\right)\end{array}$ & $\begin{array}{c}1.26 \times 10^{-5} \\
\left(0.05 \times 10^{-5}\right)\end{array}$ \\
\hline
\end{tabular}


Table 2

\section{Estimates of the Risk-Neutral Drift Parameters}

\begin{tabular}{|c|ccc|}
\hline & SQRT & CEV & 2GAM \\
Parameter & $1988-2000$ & $1988-2000$ & $1988-2000$ \\
\hline$\alpha^{*} \times 10^{6}$ & 3.006 & 0.064 & 0.817 \\
$\beta^{*}$ & 0.014 & 0.067 & 0.090 \\
\hline
\end{tabular}


Table 3

\section{Distributions of Sample Skewness and Kurtosis \\ 13-Year Sample}

Distributions of sample moments implied by each model's posterior are computed from five thousand 13-year long simulated paths of prices and instantaneous variances. The parameter values used for each simulation are drawn at random from the posterior distributions from the 1988-2000 sample. The initial values of instantaneous variance used in the simulations are randomly drawn from the stationary distribution implied by each model and its corresponding parameter vector.

\begin{tabular}{|c|c|c|c|c|c|c|c|}
\hline & & \multicolumn{2}{|c|}{ Daily } & \multicolumn{2}{|c|}{ Weekly } & \multicolumn{2}{|c|}{ Monthly } \\
\hline & & Skewness & Kurtosis & Skewness & Kurtosis & Skewness & Kurtosis \\
\hline \multicolumn{8}{|c|}{ Actual Sample Moments } \\
\hline Post-Crash Sam & 1988-2000) & -0.300 & 8.06 & -0.260 & 4.76 & 0.160 & 4.19 \\
\hline Whole Sample ( & $-2000)$ & -2.231 & 49.23 & -1.405 & 17.09 & -0.881 & 8.55 \\
\hline \multicolumn{8}{|c|}{ Model-Implied Sample Moment Distributions } \\
\hline Model & Percentile & & & & & & \\
\hline \multirow[t]{5}{*}{ SQRT } & $1^{s t}$ & -0.307 & 3.83 & -0.675 & 3.38 & -1.191 & 2.69 \\
\hline & $5^{t h}$ & -0.228 & 4.02 & -0.492 & 3.64 & -0.879 & 2.98 \\
\hline & $50^{t h}$ & -0.071 & 4.63 & -0.160 & 4.45 & -0.305 & 3.93 \\
\hline & $95^{t h}$ & 0.071 & 5.70 & 0.141 & 5.95 & 0.243 & 6.18 \\
\hline & $99^{t h}$ & 0.134 & 6.40 & 0.276 & 7.12 & 0.519 & 8.30 \\
\hline \multirow[t]{5}{*}{ CEV } & $1^{s t}$ & -1.481 & 3.88 & -2.674 & 3.46 & -3.245 & 2.77 \\
\hline & $5^{\text {th }}$ & -0.578 & 4.25 & -1.165 & 3.84 & -1.694 & 3.14 \\
\hline & $50^{t h}$ & -0.039 & 7.14 & -0.126 & 6.42 & -0.287 & 5.05 \\
\hline & $95^{\text {th }}$ & 0.496 & 33.55 & 0.722 & 28.26 & 0.721 & 16.99 \\
\hline & $99^{t h}$ & 1.727 & 106.19 & 2.393 & 65.23 & 1.865 & 30.66 \\
\hline \multirow[t]{5}{*}{ 2GAM } & $1^{s t}$ & -2.862 & 3.96 & -3.837 & 3.48 & -3.717 & 2.76 \\
\hline & $5^{t h}$ & -0.989 & 4.33 & -1.696 & 3.89 & -2.067 & 3.08 \\
\hline & $50^{t h}$ & -0.119 & 6.95 & -0.275 & 6.07 & -0.456 & 4.78 \\
\hline & $95^{t h}$ & 0.139 & 27.80 & 0.168 & 21.38 & 0.195 & 15.10 \\
\hline & $99^{t h}$ & 0.336 & 73.34 & 0.433 & 49.03 & 0.485 & 29.99 \\
\hline \multirow[t]{5}{*}{ Pan-SVJ } & $1^{\text {st }}$ & -0.632 & 3.81 & -0.477 & 3.48 & -0.647 & 2.96 \\
\hline & $5^{t h}$ & -0.411 & 4.03 & -0.318 & 3.65 & -0.445 & 3.14 \\
\hline & $50^{t h}$ & -0.057 & 5.15 & -0.048 & 4.24 & -0.055 & 3.76 \\
\hline & $95^{\text {th }}$ & 0.251 & 9.04 & 0.217 & 5.45 & 0.352 & 5.04 \\
\hline & $99^{t h}$ & 0.480 & 12.46 & 0.347 & 6.52 & 0.566 & 6.35 \\
\hline
\end{tabular}


Table 4

\section{Distributions of Sample Skewness and Kurtosis \\ 115-Year Sample}

Distributions of sample moments implied by each model's posterior are computed from five thousand 115-year long simulated paths of prices and instantaneous variances. The parameter values used for each simulation are drawn at random from the posterior distributions from the 1988-2000 sample. The initial values of instantaneous variance used in the simulations are randomly drawn from the stationary distribution implied by each model and its corresponding parameter vector.

\begin{tabular}{|c|c|c|c|c|c|c|c|}
\hline & \multicolumn{2}{|c|}{ Daily } & \multicolumn{2}{|c|}{ Weekly } & \multicolumn{2}{|c|}{ Monthly } \\
\hline & & Skewness & Kurtosis & Skewness & Kurtosis & Skewness & Kurtosis \\
\hline \multicolumn{8}{|c|}{ Actual Sample Moments } \\
\hline Extended Sampl & $1885-2000)$ & -0.157 & 22.63 & -0.184 & 11.72 & 0.252 & 12.71 \\
\hline \multicolumn{8}{|c|}{ Model-Implied Sample Moment Distributions } \\
\hline Model & Percentile & & & & & & \\
\hline SQRT & $\begin{array}{r}1^{\text {st }} \\
5^{\text {th }} \\
50^{t h} \\
95^{t h} \\
99^{t h}\end{array}$ & $\begin{array}{r}-0.162 \\
-0.136 \\
-0.076 \\
-0.019 \\
0.008\end{array}$ & $\begin{array}{l}4.28 \\
4.40 \\
4.80 \\
5.41 \\
5.76\end{array}$ & $\begin{array}{r}-0.376 \\
-0.310 \\
-0.178 \\
-0.054 \\
0.002\end{array}$ & $\begin{array}{l}4.09 \\
4.23 \\
4.72 \\
5.51 \\
5.99\end{array}$ & $\begin{array}{r}-0.677 \\
-0.569 \\
-0.334 \\
-0.091 \\
0.045\end{array}$ & $\begin{array}{l}3.61 \\
3.82 \\
4.44 \\
5.53 \\
6.25\end{array}$ \\
\hline $\mathrm{CEV}$ & $\begin{array}{r}1^{\text {st }} \\
5^{t h} \\
50^{t h} \\
95^{t h} \\
99^{t h}\end{array}$ & $\begin{array}{r}-3.033 \\
-0.803 \\
-0.051 \\
1.577 \\
7.183\end{array}$ & $\begin{array}{r}5.44 \\
6.72 \\
17.79 \\
191.35 \\
684.71\end{array}$ & $\begin{array}{r}-4.122 \\
-1.620 \\
-0.200 \\
1.528 \\
7.328\end{array}$ & $\begin{array}{r}5.07 \\
6.10 \\
14.46 \\
107.31 \\
311.05\end{array}$ & $\begin{array}{r}-3.464 \\
-2.039 \\
-0.448 \\
1.065 \\
4.892\end{array}$ & $\begin{array}{r}4.39 \\
5.12 \\
10.35 \\
47.64 \\
127.32\end{array}$ \\
\hline 2GAM & $\begin{array}{r}1^{\text {st }} \\
5^{t h} \\
50^{t h} \\
95^{t h} \\
99^{t h}\end{array}$ & $\begin{array}{r}-4.208 \\
-1.526 \\
-0.251 \\
-0.033 \\
0.125\end{array}$ & $\begin{array}{r}5.60 \\
6.61 \\
12.30 \\
60.85 \\
210.42\end{array}$ & $\begin{array}{l}-4.039 \\
-2.108 \\
-0.539 \\
-0.161 \\
-0.035\end{array}$ & $\begin{array}{r}4.98 \\
5.84 \\
10.22 \\
43.82 \\
99.99\end{array}$ & $\begin{array}{l}-3.368 \\
-2.217 \\
-0.822 \\
-0.312 \\
-0.138\end{array}$ & $\begin{array}{r}4.30 \\
4.85 \\
8.09 \\
24.86 \\
44.41\end{array}$ \\
\hline Pan-SVJ & $\begin{array}{r}1^{s t} \\
5^{t h} \\
50^{t h} \\
95^{t h} \\
99^{t h}\end{array}$ & $\begin{array}{r}-0.244 \\
-0.188 \\
-0.068 \\
0.044 \\
0.099\end{array}$ & $\begin{array}{l}4.66 \\
4.89 \\
5.66 \\
6.99 \\
7.67\end{array}$ & $\begin{array}{r}-0.190 \\
-0.148 \\
-0.053 \\
0.041 \\
0.078\end{array}$ & $\begin{array}{l}4.01 \\
4.11 \\
4.41 \\
4.83 \\
5.09\end{array}$ & $\begin{array}{r}-0.261 \\
-0.202 \\
-0.059 \\
0.084 \\
0.159\end{array}$ & $\begin{array}{l}3.54 \\
3.66 \\
3.98 \\
4.47 \\
4.76\end{array}$ \\
\hline
\end{tabular}


Table 5

\section{Distributions of Crash Frequencies}

\section{5-Year Sample}

Distributions of crash frequencies implied by each model's posterior are computed from five thousand 115-year long simulated paths of prices and instantaneous variances. A crash frequency is the number of times in a single sample that a return falls below some prespecified level, either $-5,-10$, or -20 percent. The parameter values used for each simulation are drawn at random from the posterior distributions from the 1988-2000 sample. The initial values of instantaneous variance used in the simulations are randomly drawn from the stationary distribution implied by each model and its corresponding parameter vector.

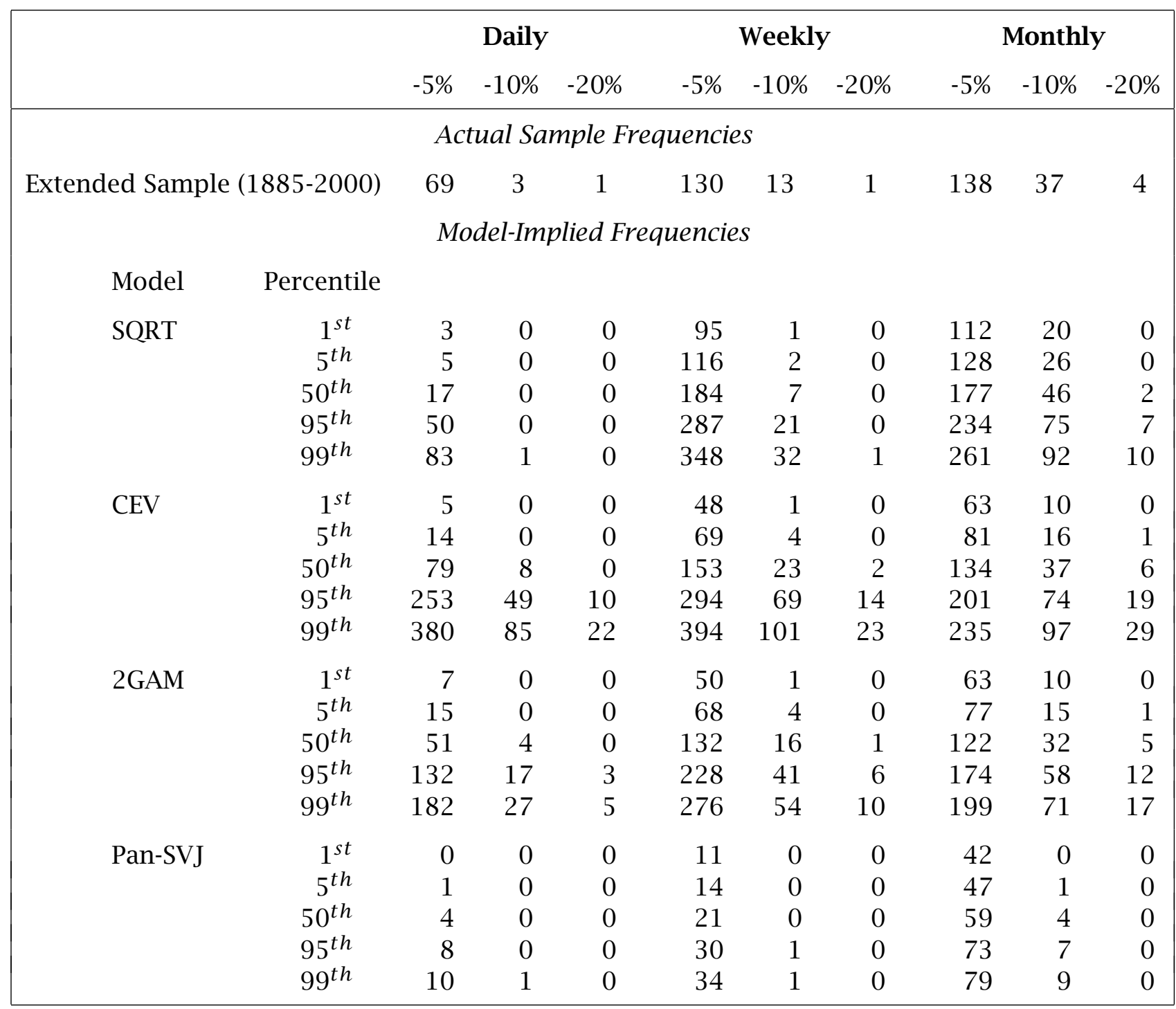


Table 6

\section{Model-Implied Put and Call Prices}

Option prices are calculated numerically using parameter values set equal to the 1988-2000 posterior means reported in Table 1. The initial stock price is 100 and the interest rate is zero.

\begin{tabular}{|c|c|c|c|c|c|c|c|c|c|c|}
\hline & \multirow[b]{2}{*}{ Strike } & \multicolumn{4}{|c|}{ Puts } & ATM & \multicolumn{4}{|c|}{ Calls } \\
\hline & & 80 & 90 & 95 & 99 & 100 & 101 & 105 & 110 & 120 \\
\hline \multirow{3}{*}{$\begin{array}{l}\tau=5 \\
V_{0}=.0001\end{array}$} & SQRT & 0.0000 & 0.0001 & 0.0250 & 0.5399 & 0.9405 & 0.5050 & 0.0069 & 0.0000 & 0.0000 \\
\hline & CEV & 0.0000 & 0.0001 & 0.0289 & 0.5642 & 0.9686 & 0.5344 & 0.0102 & 0.0000 & 0.0000 \\
\hline & 2GAM & 0.0000 & 0.0004 & 0.0389 & 0.6004 & 1.0035 & 0.5629 & 0.0117 & 0.0000 & 0.0000 \\
\hline \multirow{3}{*}{$\begin{array}{l}\tau=5, \\
V_{0}=.001\end{array}$} & SQRT & 0.0033 & 0.2460 & 1.0160 & 2.3998 & 2.8792 & 2.4133 & 1.0706 & 0.2994 & 0.0093 \\
\hline & CEV & 0.0299 & 0.4258 & 1.2477 & 2.5795 & 3.0343 & 2.5296 & 1.0851 & 0.2639 & 0.0039 \\
\hline & 2GAM & 0.0835 & 0.5508 & 1.3482 & 2.5976 & 3.0277 & 2.5007 & 0.9833 & 0.1757 & 0.0003 \\
\hline \multirow{3}{*}{$\begin{array}{l}\tau=22 \\
V_{0}=.0001\end{array}$} & SQRT & 0.0029 & 0.1539 & 0.6890 & 1.8309 & 2.2669 & 1.7832 & 0.5030 & 0.0469 & 0.0000 \\
\hline & CEV & 0.0330 & 0.3697 & 1.0838 & 2.3076 & 2.7416 & 2.2334 & 0.8023 & 0.1224 & 0.0002 \\
\hline & 2GAM & 0.1276 & 0.6607 & 1.4798 & 2.7194 & 3.1413 & 2.6262 & 1.0599 & 0.1994 & 0.0004 \\
\hline \multirow{3}{*}{$\begin{array}{l}\tau=22 \\
V_{0}=.001\end{array}$} & SQRT & 0.6835 & 2.5134 & 4.1886 & 5.9618 & 6.4667 & 5.9940 & 4.3507 & 2.7965 & 1.0043 \\
\hline & CEV & 2.2206 & 4.3927 & 6.0101 & 7.6201 & 8.0705 & 7.5142 & 5.6024 & 3.6873 & 1.3249 \\
\hline & 2GAM & 2.0807 & 3.9812 & 5.4304 & 6.9066 & 7.3255 & 6.8028 & 4.7889 & 2.8097 & 0.6221 \\
\hline \multirow{3}{*}{$\begin{array}{l}\tau=66 \\
V_{0}=.0001\end{array}$} & SQRT & 0.5768 & 2.0246 & 3.4298 & 5.0008 & 5.4626 & 4.9549 & 3.2108 & 1.6788 & 0.3109 \\
\hline & CEV & 4.6296 & 7.2893 & 9.0223 & 10.6370 & 11.0746 & 10.5890 & 8.5398 & 6.3168 & 3.0253 \\
\hline & 2GAM & 4.2936 & 6.9236 & 8.6442 & 10.2477 & 10.6822 & 10.2715 & 8.2119 & 5.9796 & 2.7032 \\
\hline \multirow{3}{*}{$\begin{array}{l}\tau=66 \\
V_{0}=.001\end{array}$} & SQRT & 4.9226 & 8.5359 & 10.8033 & 12.8356 & 13.3735 & 12.9174 & 11.2297 & 9.3669 & 6.3855 \\
\hline & CEV & 13.3707 & 17.4144 & 19.6474 & 21.5386 & 22.0262 & 23.5379 & 21.5729 & 19.2551 & 15.0914 \\
\hline & 2GAM & 6.7646 & 10.0521 & 12.0286 & 13.7804 & 14.2427 & 14.1387 & 12.1290 & 9.8511 & 6.0954 \\
\hline
\end{tabular}


Figure 1A: S\&P 100 Implied Volatility

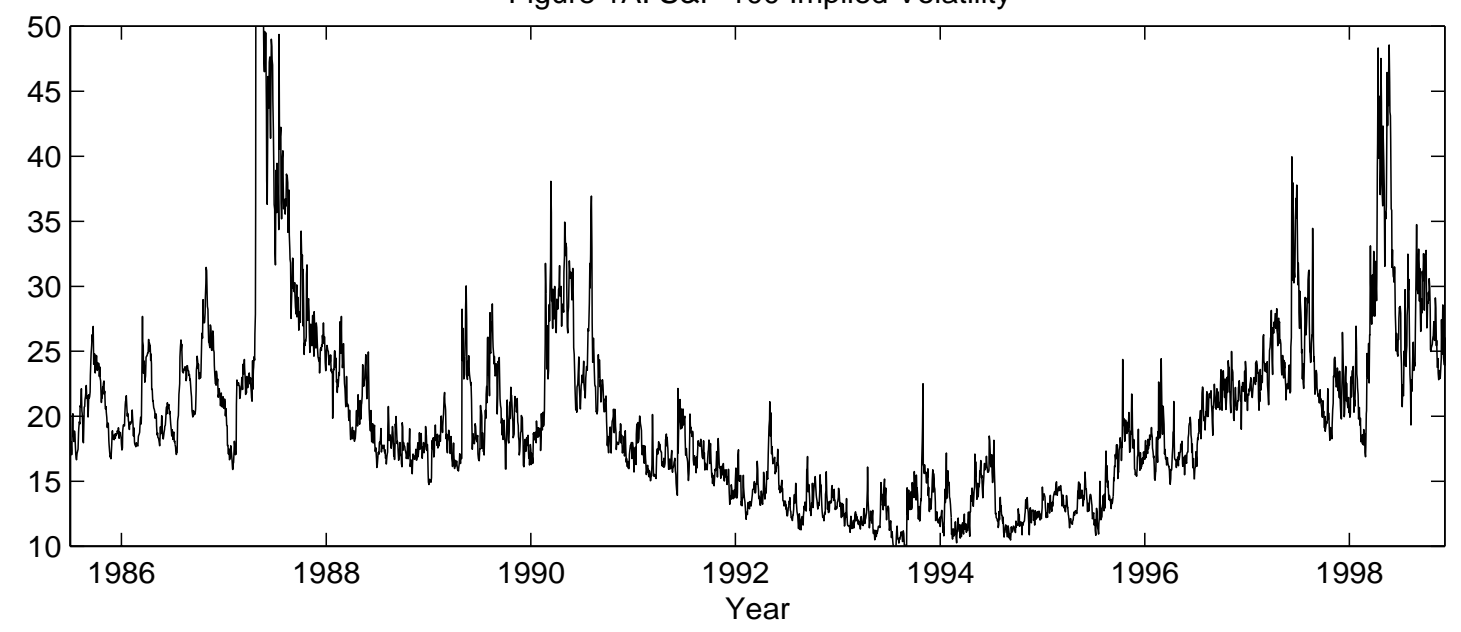

Figure 1B: Absolute Changes in Implied Volatility as a Function of its Level

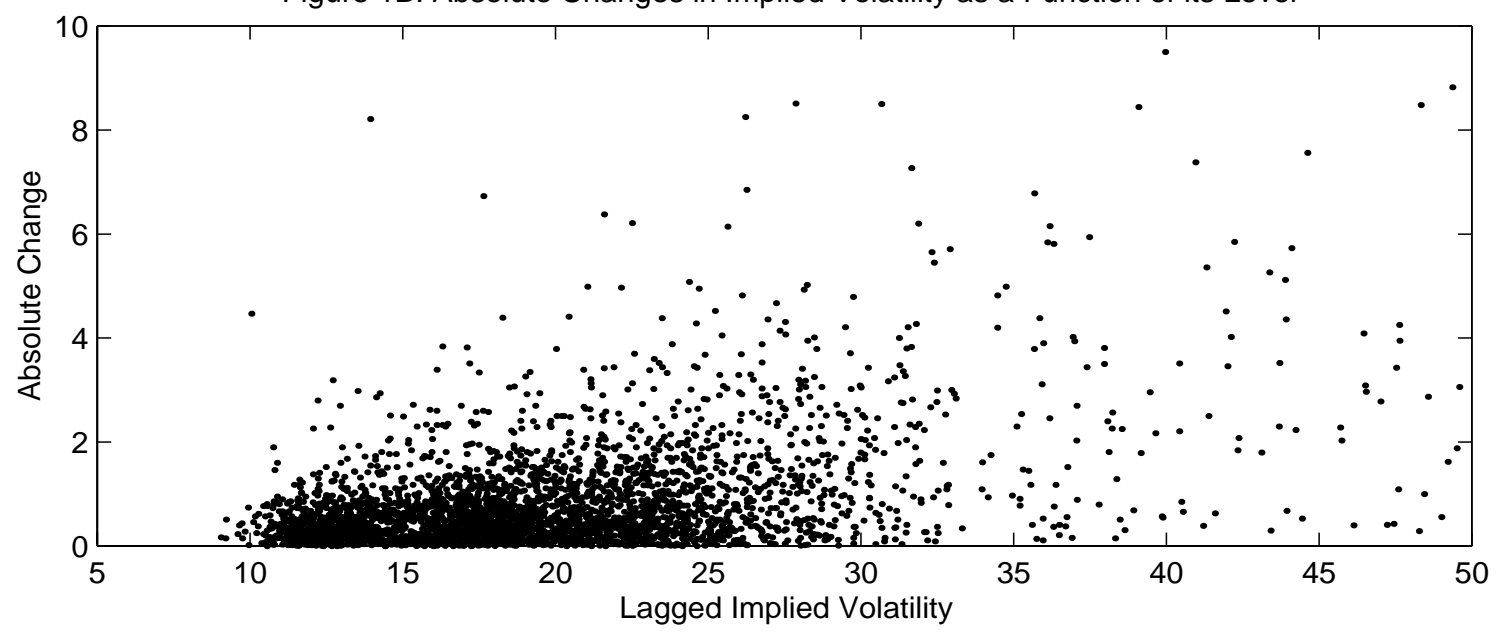


Figure 2A: Simulated Maximum Likelihood



Figure 2B: Bayesian Data Augmentation

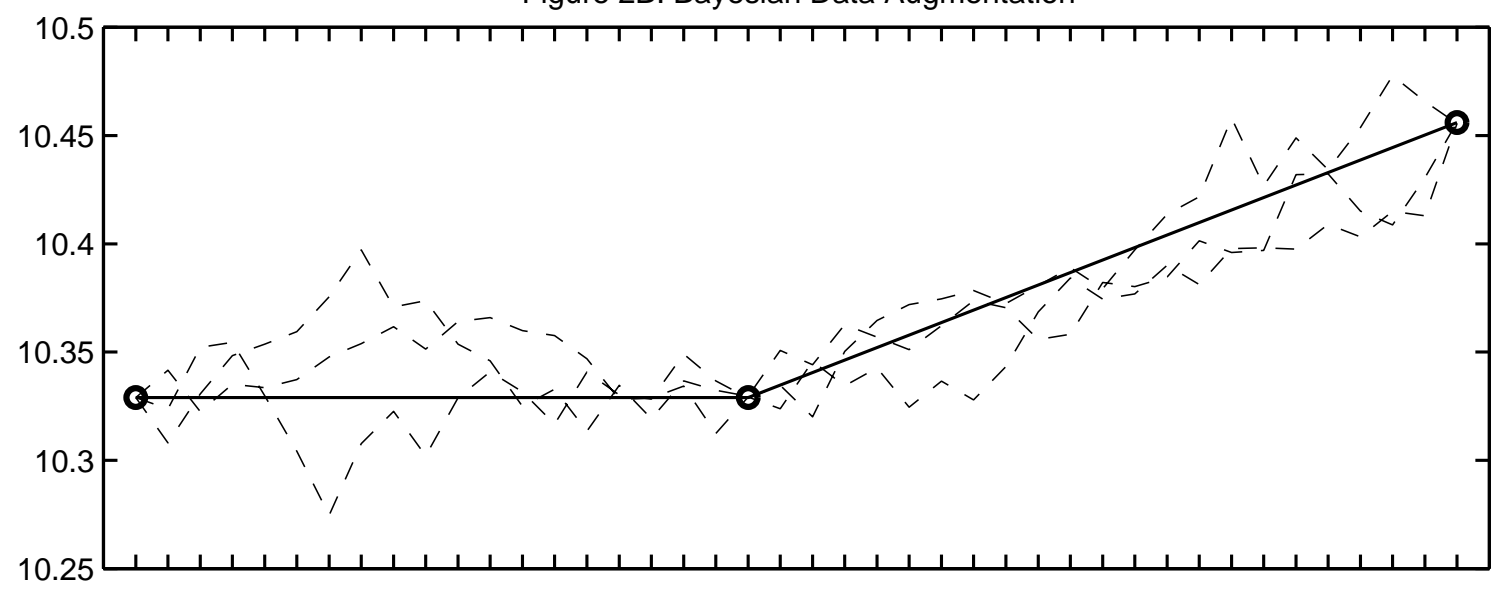


Relation Between Instantaneous and Black-Scholes Implied Variances for the SQRT Model
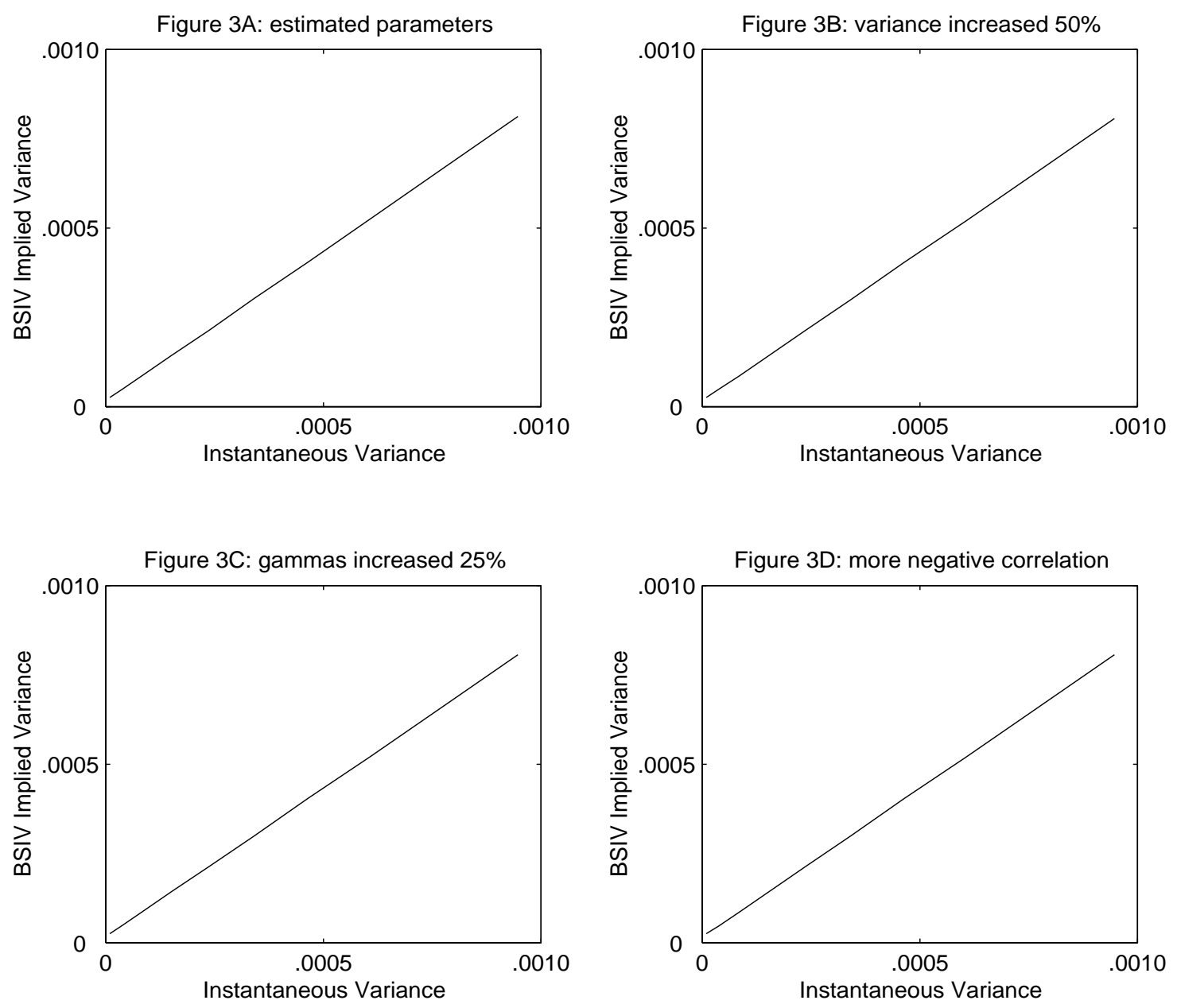
Relation Between Instantaneous and Black-Scholes Implied Variances for the CEV Model
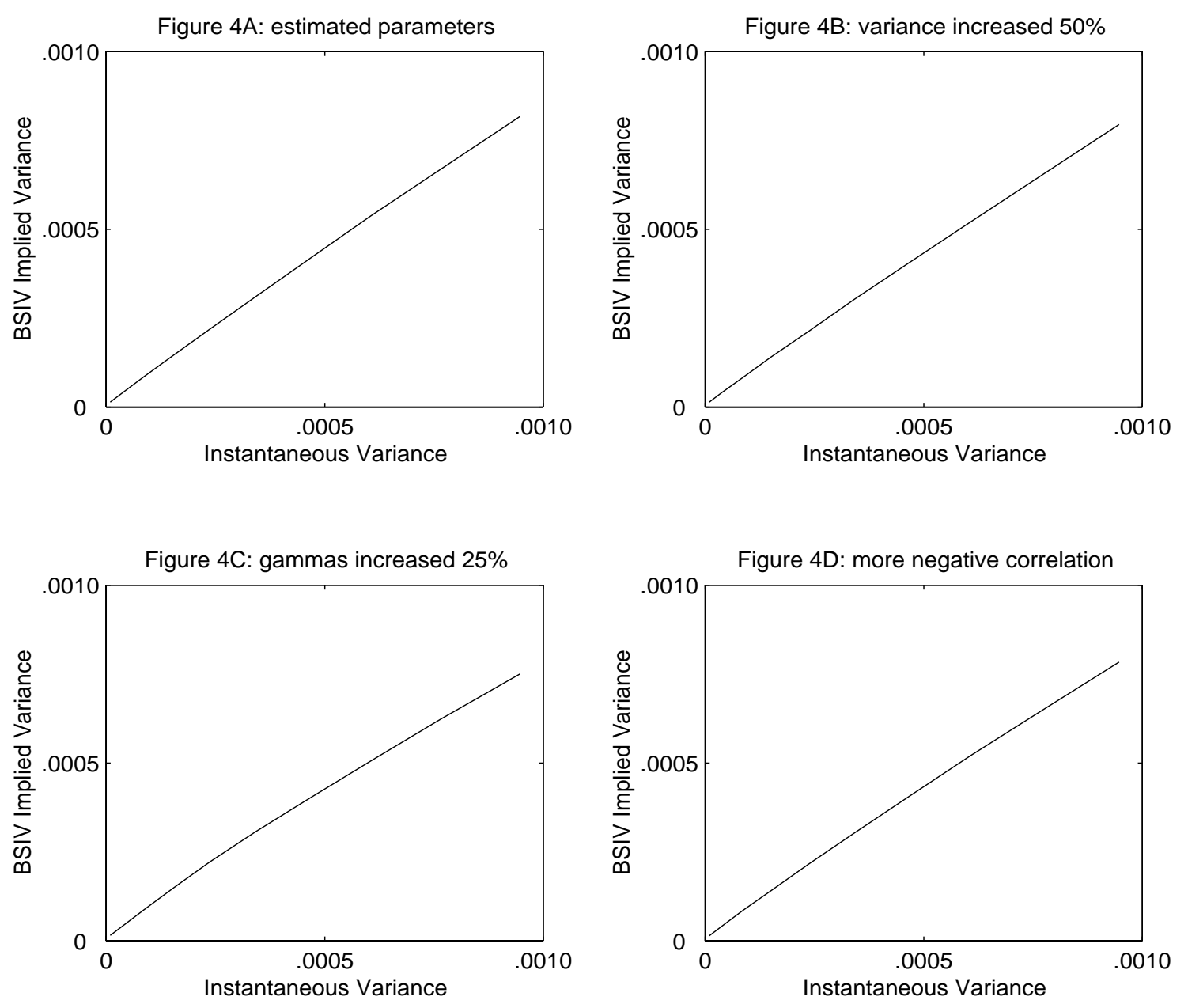
Relation Between Instantaneous and Black-Scholes Implied Variances for the 2GAM Model
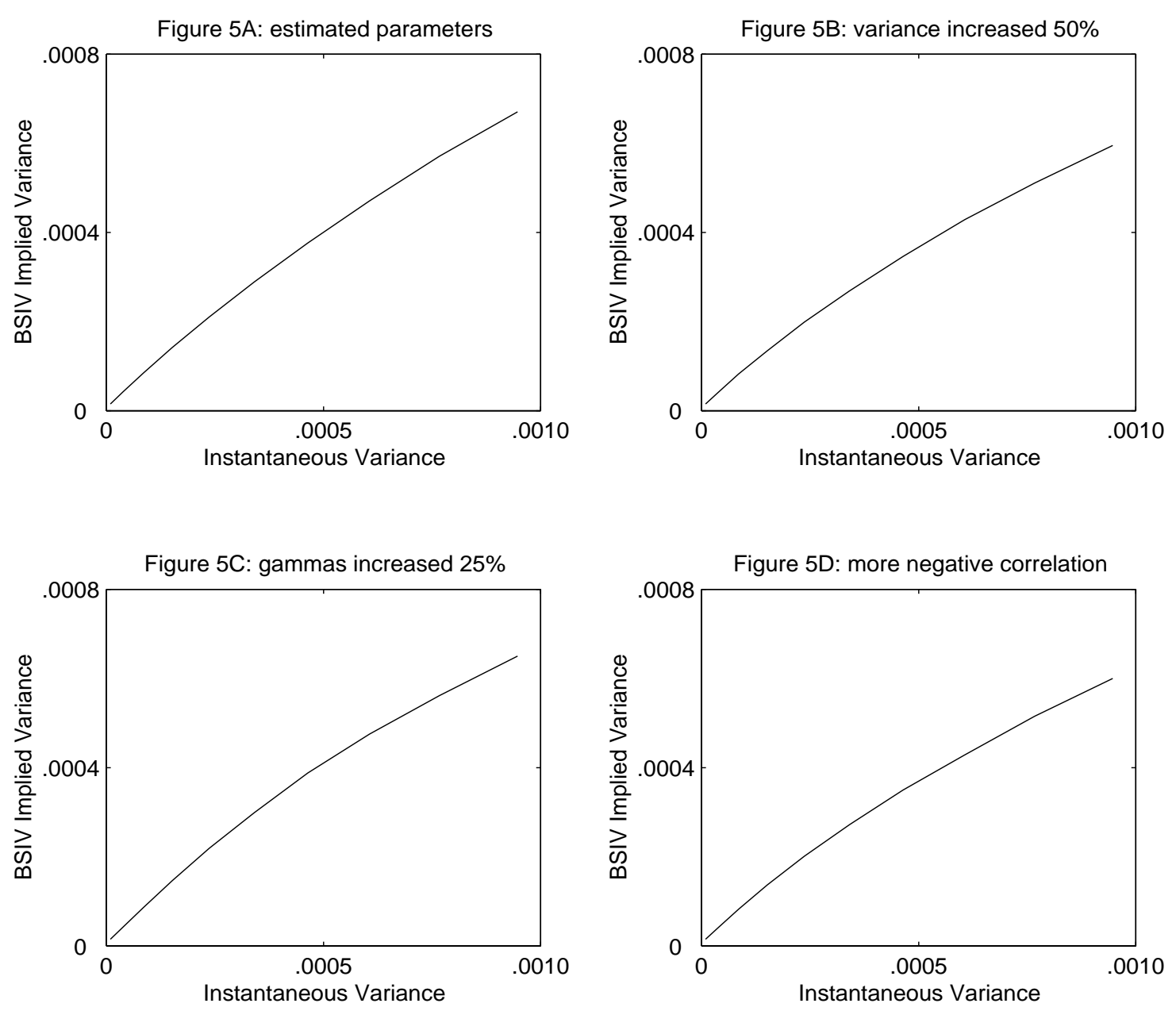
Figure 6A: SQRT steady-state distribution of instantaneous variance under true measure

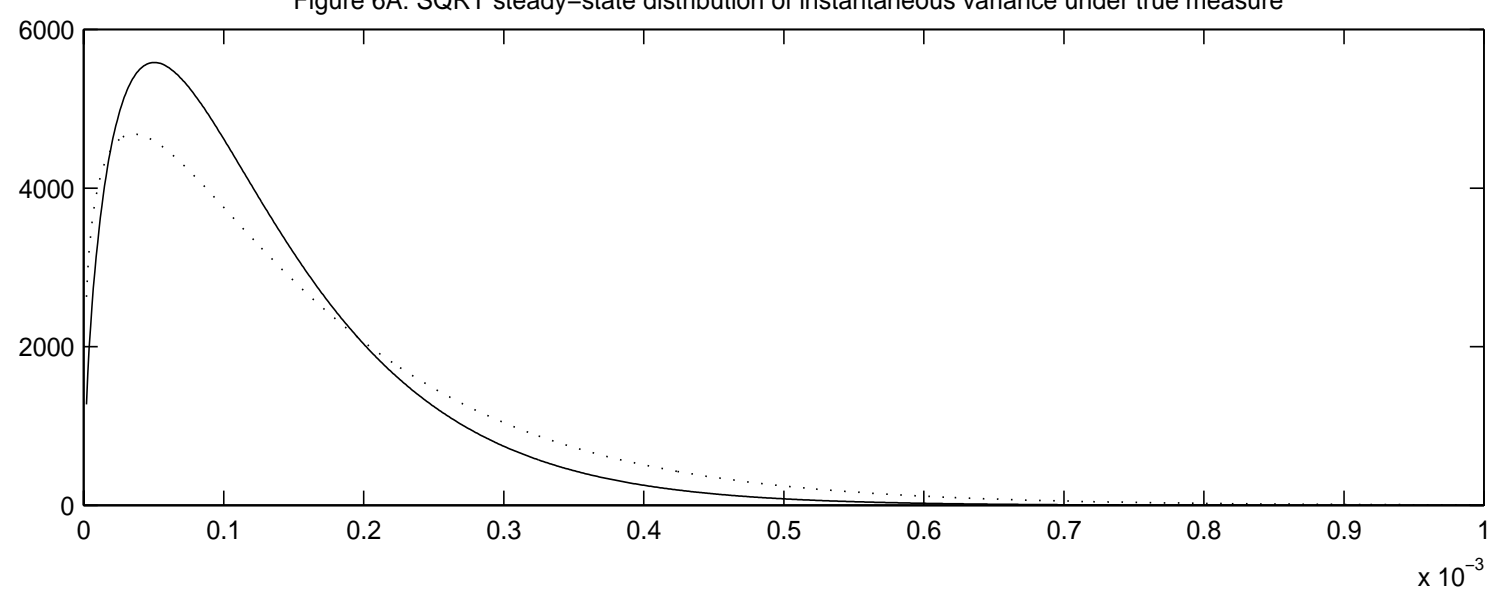

Figure 6B: CEV steady-state distribution of instantaneous variance under true measure

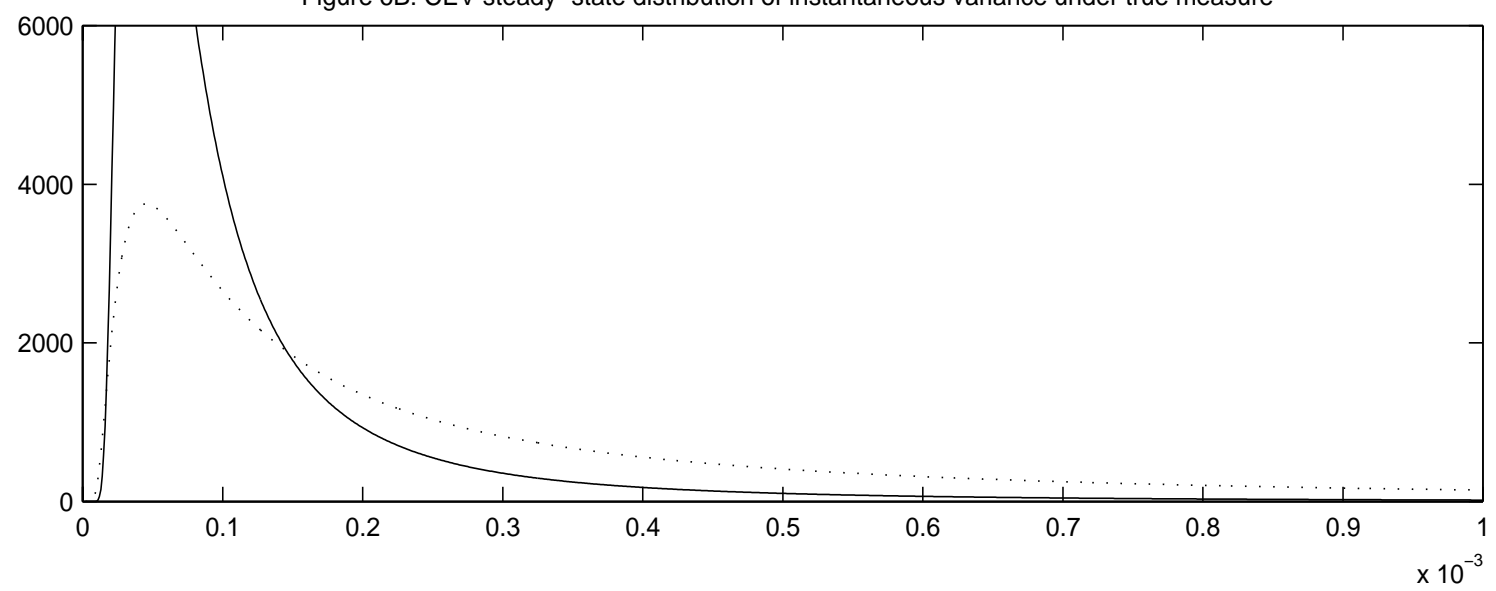

Figure 6C: VEV steady-state distribution of instantaneous variance under true measure

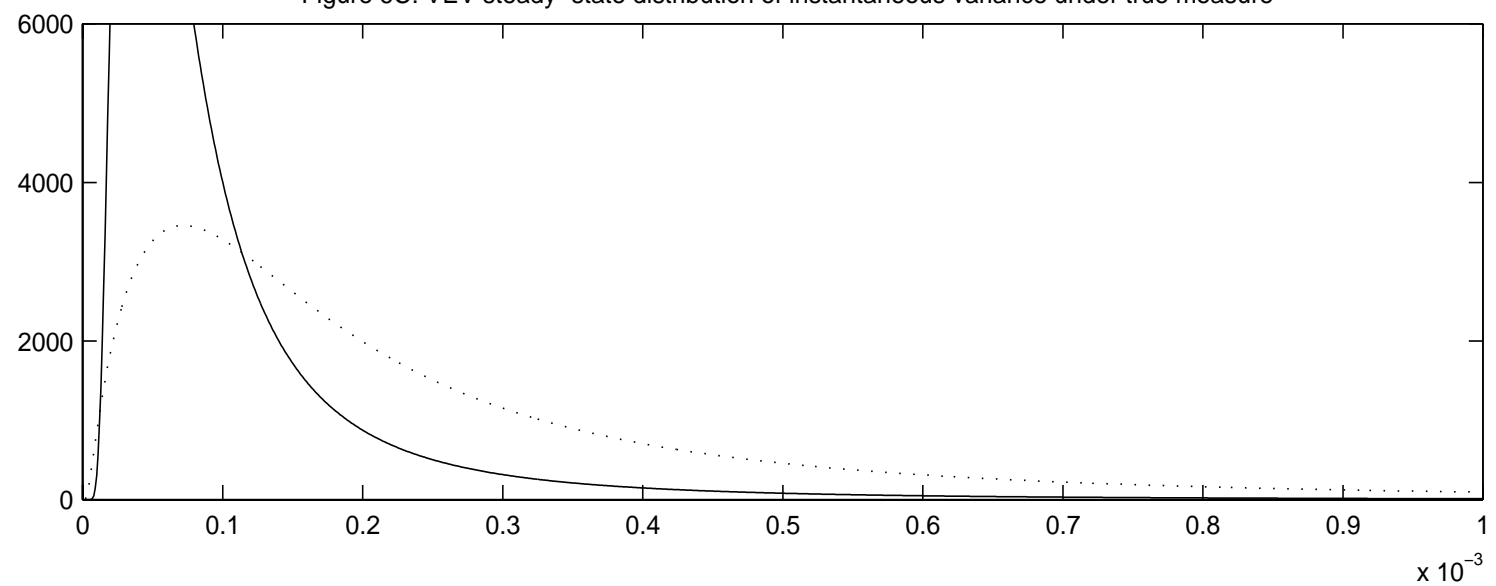

Solid lines are calculated using posterior means from July 1988 to January 2000. Dotted lines use the January 1986 to January 2000 posterior means. 
Figure 7A: ratio of CEV density to SQRT density

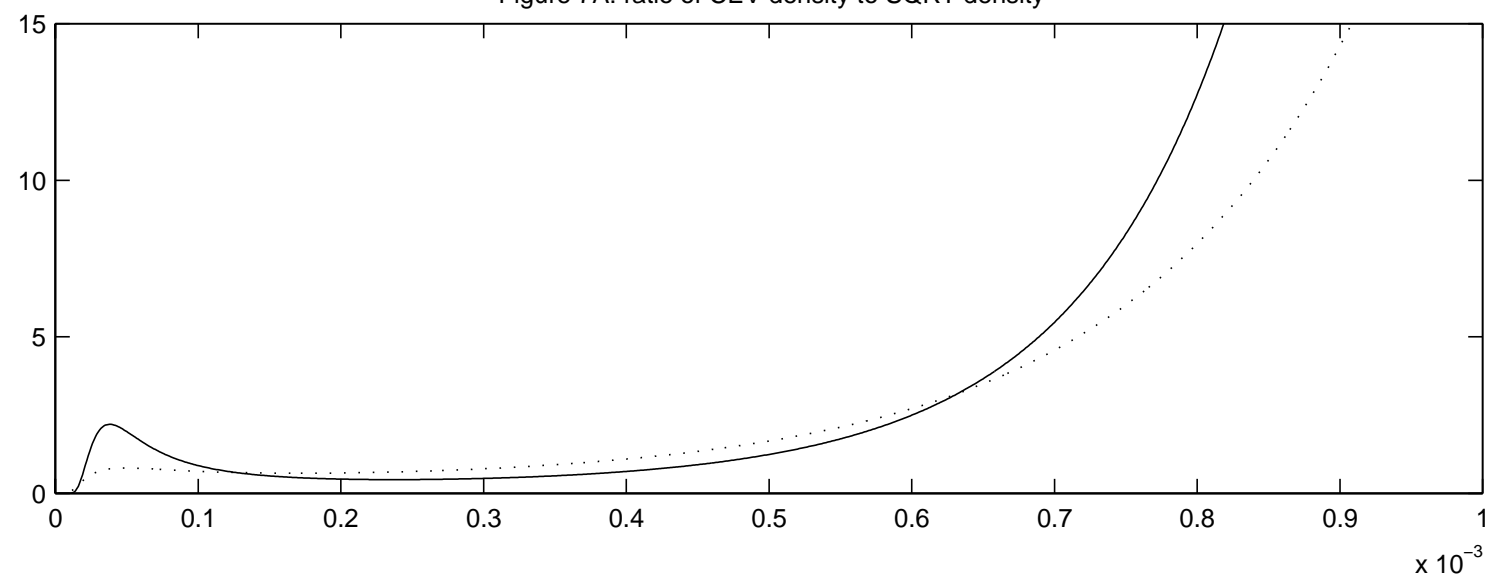

Figure 7B: ratio of VEV density to SQRT density

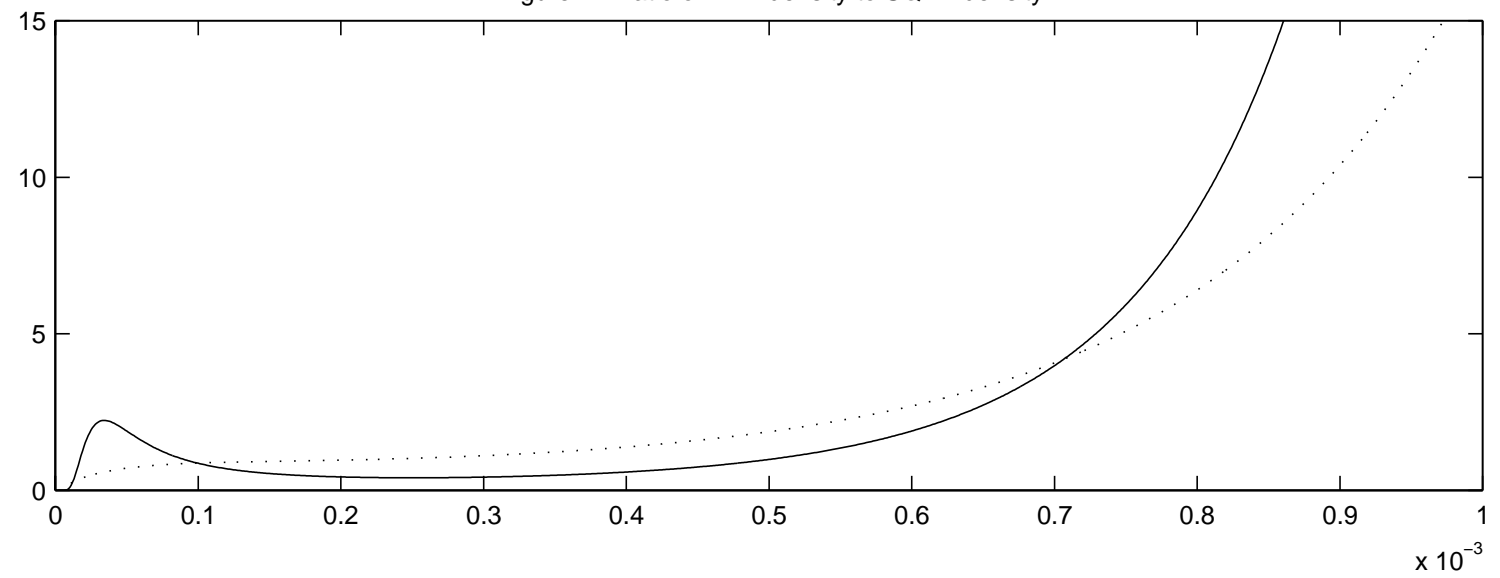

Figure 7C: ratio of VEV density to CEV density

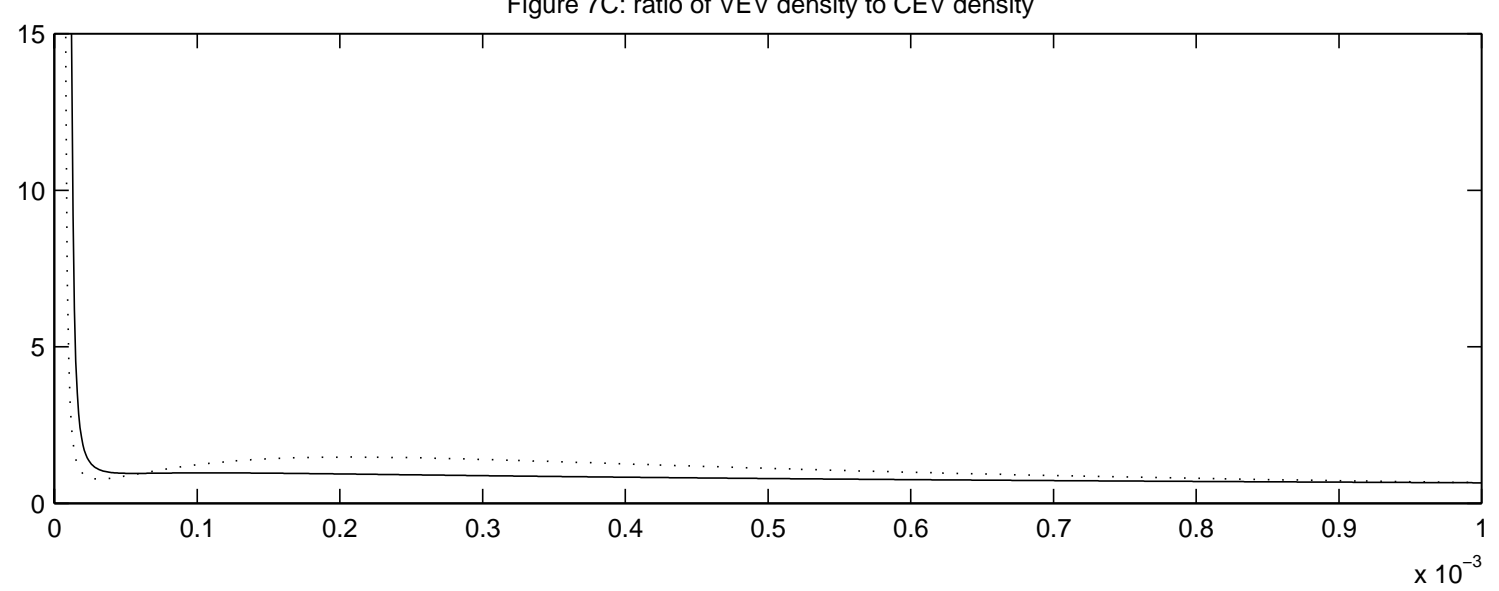

Solid lines are calculated using posterior means from July 1988 to January 2000. Dotted lines use the January 1986 to January 2000 posterior means. 
Figure 8: Simulated Volatility Paths
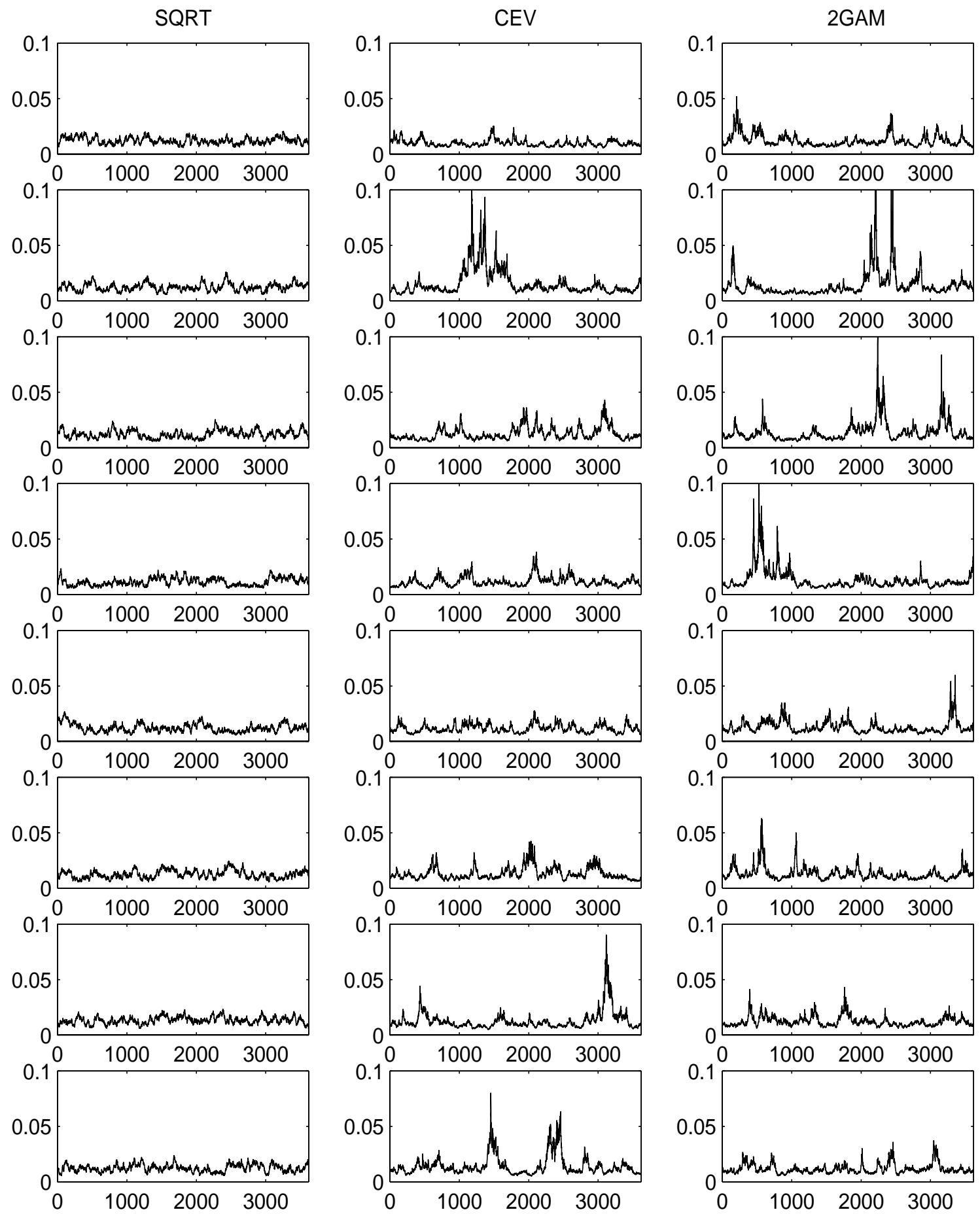
Figure 9: Correlation Between Price and Instantaneous Variance

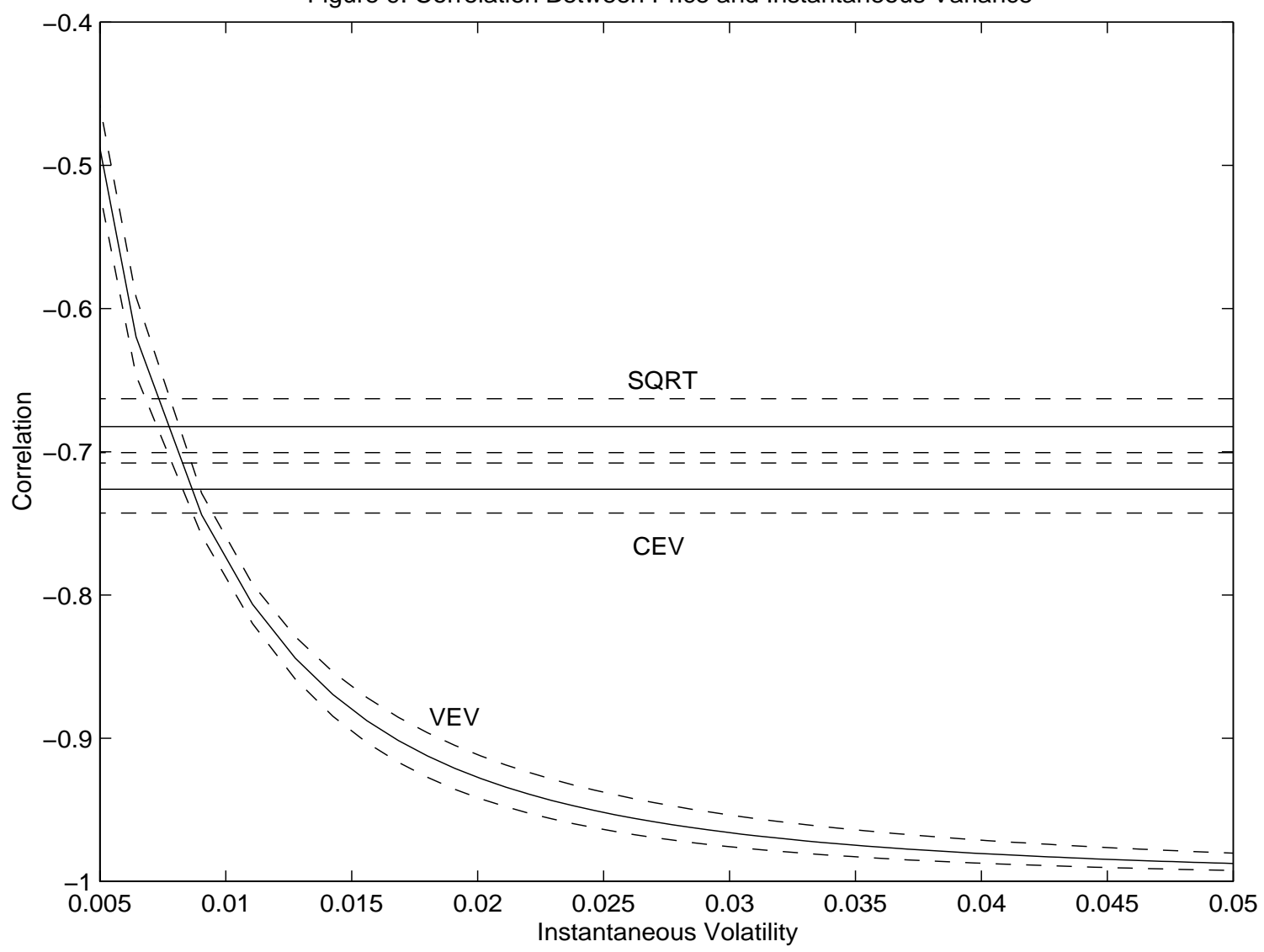

Solid lines represent posterior medians, while dashed lines denote 5th and 95th percentiles. 
Figure 10: Implied Volatility Smiles

22-day smiles, $\mathrm{V}=.0001$

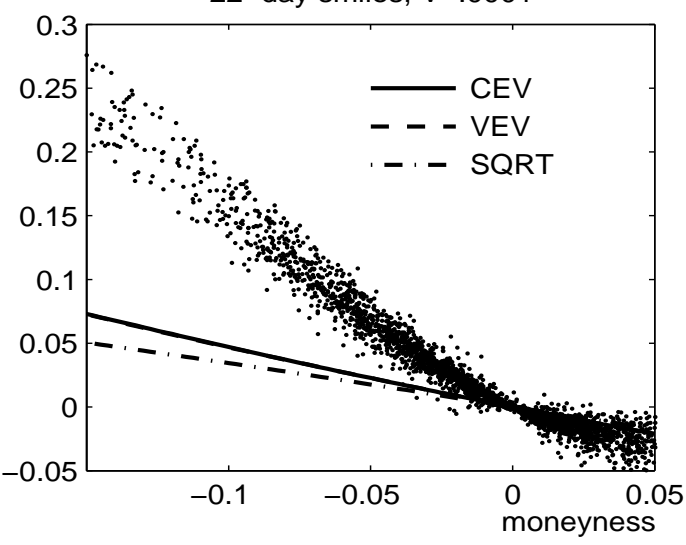

66-day smiles, $V=.0001$

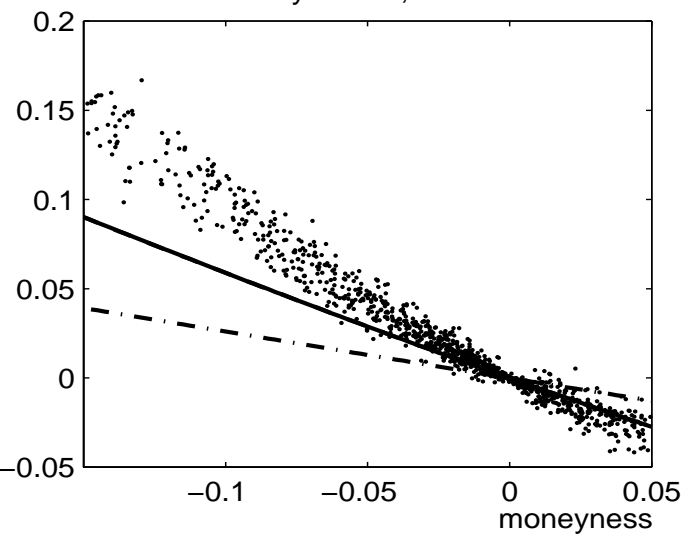

132-day smiles, $\mathrm{V}=.0001$

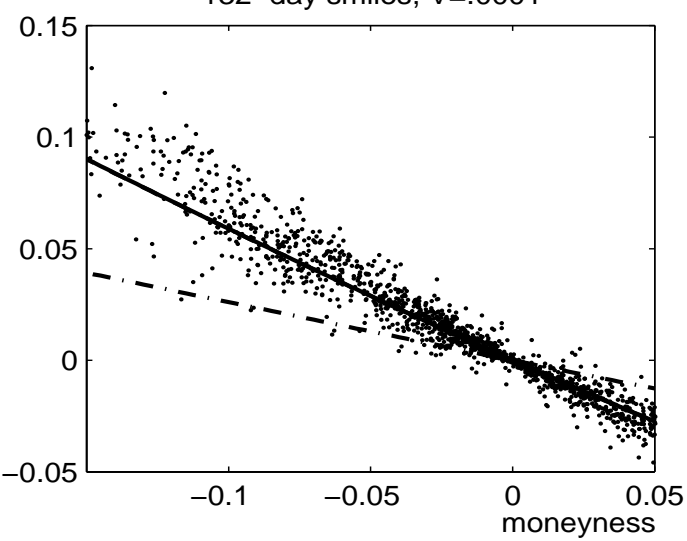

22-day smiles, $\mathrm{V}=.001$

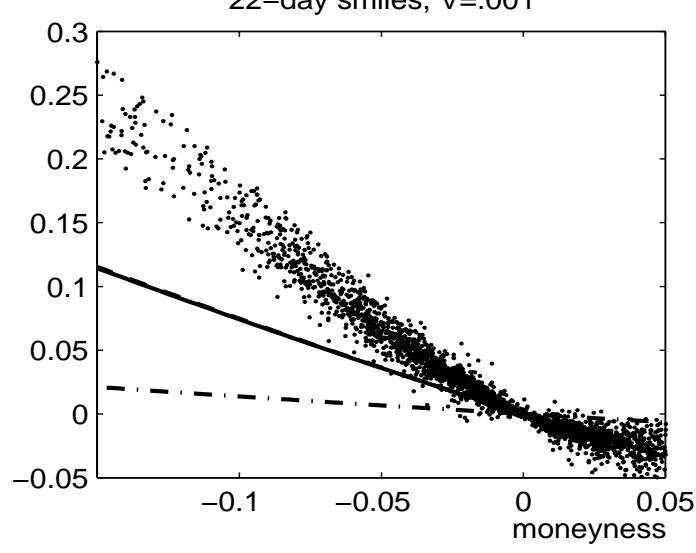

66-day smiles, $\mathrm{V}=.001$

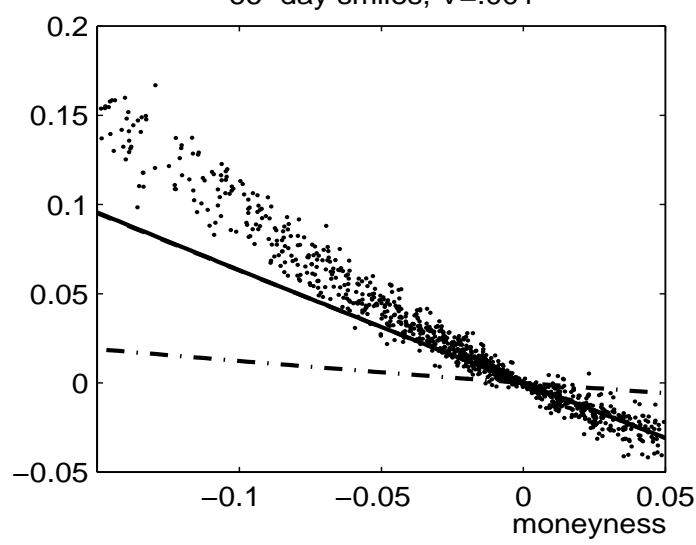

132-day smiles, $\mathrm{V}=.001$

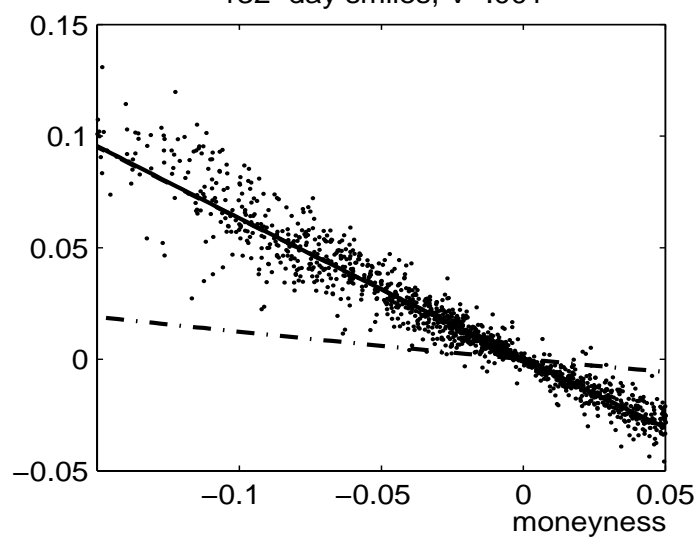

The implied volatility smile is the difference between an option's Black-Scholes implied volatility and the volatility of a contemporaneously observed at-the-money option with the same maturity. Dots represent individual observations of the smile for a particular maturity, while lines denote the volatility smiles implied by each model using the 1988-2000 posterior means. The dashed line, representing the VEV model, is not clearly visible because it is usually coincident with the solid line, representing the CEV model. 